\title{
Right Hemisphere Sensitivity to Word- and Sentence-Level Context: Evidence From Event-Related Brain Potentials
}

\author{
Seana Coulson \\ University of California, San Diego \\ Cyma Van Petten \\ University of Arizona
}

\author{
Kara D. Federmeier \\ University of Illinois \\ Marta Kutas \\ University of California, San Diego
}

\begin{abstract}
Researchers using lateralized stimuli have suggested that the left hemisphere is sensitive to sentence-level context, whereas the right hemisphere $(\mathrm{RH})$ primarily processes word-level meaning. The authors investigated this message-blind RH model by measuring associative priming with event-related brain potentials (ERPs). For word pairs in isolation, associated words elicited more positive ERPs than unassociated words with similar magnitudes and onset latencies in both visual fields. Embedded in sentences, these same pairs showed large sentential context effects in both fields. Small effects of association were observed, confined to incongruous sentences after right visual hemifield presentation but present for both congruous and incongruous sentences after left visual hemifield presentation. Results do not support the message-blind RH model but do suggest hemispheric asymmetries in the use of word and sentence context during real-time processing.
\end{abstract}

Left-hemisphere ( $\mathrm{LH})$ dominance for language is probably the most well-known instance of hemispheric asymmetry in humans. Patients with left-hemisphere damage often present with severe difficulties in the most basic aspects of language production and comprehension. However, the right hemisphere, although not as essential as the left for these basic language functions, also plays a role in language comprehension, as patients with righthemisphere damage exhibit subtle deficits in comprehending the relationship between an utterance and its context (Joanette, Goulet, \& Hannequin, 1990). Right-hemisphere patients, for example, have difficulty understanding certain kinds of jokes, display overly literal interpretation of metaphoric language, and have difficulty interpreting sarcastic utterances (McDonald, 1996).

Right-hemisphere contribution to language comprehension has also been tested in neurologically intact individuals via the use of lateralized stimuli. This paradigm involves presenting stimuli in either the left or the right visual hemifield (LVF or RVF), taking

Seana Coulson, Department of Cognitive Science, University of California, San Diego; Kara D. Federmeier, Department of Psychology, University of Illinois; Cyma Van Petten, Department of Psychology, University of Arizona; Marta Kutas, Departments of Cognitive Science and Neurosciences, University of California, San Diego.

The research reported herein was supported by Grant AG08313 from the National Institute of Aging and Grant HD22614 from the National Institute of Child Health and Human Development to Marta Kutas and by a research grant from the University of California, San Diego Academic Senate to Seana Coulson. We thank Esmeralda DeOchoa, Etienne Korvorst, Marcus Lauer, and Marjolein Meeuwissen for their help with data collection.

Correspondence concerning this article should be addressed to Seana Coulson, Cognitive Science 0515, 9500 Gilman Drive, La Jolla, CA 92093-0515. E-mail: coulson@cogsci.ucsd.edu advantage of the fact that stimuli presented outside of the center of gaze are initially processed only by the contralateral hemisphere (Hellige, 1983; Zaidel, 1983). Although information presented in this manner can be rapidly transmitted to both hemispheres, the hemifield technique is thought to reveal initial hemisphere-specific computations (Chiarello, 1991). As a consequence, left visual field presentation is typically abbreviated as $\mathrm{LVF} / \mathrm{rh}$, whereas right visual field presentation is abbreviated $\mathrm{RVF} / \mathrm{lh}$.

Hemifield language research in healthy adults suggests the two cerebral hemispheres differ in the way they establish word meaning (Chiarello, 1988). For example, although most studies using strongly associated pairs (cat-dog) report equivalent context effects with RVF and LVF presentation, nonassociated category members such as goat and dog yield stronger effects with LVF/rh presentation (Chiarello, Burgess, Richards, \& Pollock, 1990). Larger effects after LVF/rh presentation have been observed for the subordinate meanings of ambiguous words (Burgess \& Simpson, 1988; Titone, 1998). In addition to such differences in semantic representation (Beeman \& Chiarello, 1998) and/or the spread of semantic activation (Burgess \& Lund, 1998; Burgess \& Simpson, 1988; Koivisto \& Laine, 2000), researchers have argued for very different sentence processing mechanisms in the two hemispheres.

Faust and colleagues, for example, maintain that although the LH has the ability to integrate syntactic, semantic, and pragmatic information to construct a message-level representation of meaning, RH language competence extends only to word-level priming mechanisms (Faust, 1998; Faust, Babkoff, \& Kravets, 1995; Faust \& Gernsbacher, 1996; Faust, Kravetz, \& Babkoff, 1993). For example, in a comparison of lexical decision latencies for words such as horse when presented in normal or scrambled sentences, normal sentences yielded substantially faster reaction times (RTs) 
when presented to the RVF/lh but not the LVF/rh (Faust et al., 1995). Likewise, increasing the amount of context from one to three to six words yielded larger priming effects with presentation to the RVF/lh but not the LVF/rh (Faust et al., 1993). Further, $\mathrm{RVF} / \mathrm{lh}$ but not $\mathrm{LVF} / \mathrm{rh}$ presentation yielded longer lexical decision times for sentence-final words in implausible than it did for those in plausible sentences (e.g., This restaurant serves French fries with ketchup/cream. Faust, 1998).

These findings are hard to reconcile with reports of discourse comprehension deficits after right-hemisphere damage. Whereas researchers working with patients have suggested that the $\mathrm{RH}$ operates primarily at the message level, those working with healthy adults have suggested the RH is not sensitive to messagelevel meanings. Further, recent results using the hemifield paradigm have been somewhat equivocal on the extent of RH sensitivity to some aspects of sentence context. For instance, although Faust et al. (1995) observed sentence congruity effects in reaction times only after $\mathrm{RVF} / \mathrm{lh}$ presentation, lexical decision accuracies were sensitive to congruity in both visual fields. To more directly assess the sensitivity of each hemisphere to word- versus sentencelevel context, Chiarello and colleagues compared lexical decision latencies for the final words of congruous and incongruous sentences with a lexical associate (The weary campers set up/devoured their tent.), and those without a lexical associate (The weary husband set up/devoured the tent.; Chiarello, Liu, \& Faust, 2001). When lexical associates were present, facilitation for congruous targets was observed in both visual fields (VFs), albeit larger facilitation with presentation to the LVF/rh; when lexical associates were absent, inhibition for incongruous targets was observed in both VFs, but no facilitation was observed for congruous targets (both relative to a neutral condition). These results are inconsistent with the claim that the RH is sensitive only to word-level information (Faust, 1998).

Likewise, Faust and colleagues compared lexical decision times for laterally presented target words in a variety of contexts with or without a lexical associate (Faust, Bar-lev, \& Chiarello, 2003). In congruent and incongruent sentence contexts, presentation to both VFs yielded a similar pattern of effects, though priming effects were smaller with $\mathrm{LVF} / \mathrm{rh}$ presentation. It is interesting to note that when associatively primed targets occurred in syntactic prose (The store jumped from the sick child to the doctor,) priming effects were observed only with $\mathrm{LVF} / \mathrm{rh}$ and not $\mathrm{RVF} / \mathrm{lh}$ presentation. These data suggest that although the RH may indeed be sensitive to message-level congruity, associative priming mechanisms operate in a more context-blind manner in the $\mathrm{RH}$ than they do in the LH.

In many previous studies of the relative impact of word- and sentence-level context mechanisms, the factors of association, sentence congruity, cloze probability, and sentence constraint have been confounded. For example, the number of people who would produce tent as the best completion is higher when campers is the subject of the sentence than when husband is the subject of the sentence, so that the addition of a lexical associate to the sentence alters the cloze probability of the final word as well. In this study, we take a different approach to this issue by manipulating lexical association independent of cloze probability and by recording electrical brain activity (event-related potentials; ERPs) as healthy adults read laterally presented target words.

\section{Event-Related Potentials and Cognitive Processing}

ERPs provide a continuous record of stimulus processing that offers a different window into right-hemisphere language competence. In particular, ERPs provide temporally and functionally specific measures of processing that may help circumvent limitations of the lexical decision and naming tasks that have been widely used to investigate language comprehension asymmetries. For instance, research with aphasic individuals suggests that the processes that support lexical decision and those that allow semantic classification are nonidentical (Bub \& Arguin, 1995). Moreover, the speed and accuracy of lexical decision and naming often underestimate semantic processing capacity in the isolated right hemisphere of commisurotomy patients (Zaidel, 1990). These tasks thus may not fully reflect the sensitivity of the RH to the developing representation of sentence meaning at the message level.

Psychophysiologists have studied the ERPs elicited in a range of cognitive tasks for nearly 40 years and have identified a number of components known to be correlated with various perceptual, memory, and language processing operations (Rugg \& Coles, 1995). For example, the visual N1 component, an early negativity evident over posterior scalp sites, has been linked to early visual processing. Its amplitude is closely correlated with changes in participants' success in signal-detection tasks. The P2 component is a positive-going waveform thought to index feature detection, selective attention, and other aspects of perceptual encoding (Dunn, Dunn, Languis, \& Andrews, 1998). The late positive complex (LPC) is a positive-going deflection in the waveform observed 500-900 ms after stimulus onset that has typically been linked to memory processes. In language research, it has been argued that the LPC reflects semantic encoding, including elaborative processes based on information in long-term memory, and the memory demands of building a mental model of events described in a text (Van Petten, Kutas, Kluender, Mitchiner, \& McIsaac, 1991).

The N400, a negative-going component peaking at around 400 ms after stimulus onset, is of particular interest, as it has been associated with the processing of potentially meaningful events (Nobre \& McCarthy, 1995; Van Petten, 1995). In general, N400 amplitude is seen as an index of the difficulty of integrating a word into a given context: the larger the N400, the more difficult the task of lexical-semantic integration (Kutas, Federmeier, Coulson, King, \& Munte, 2000). Words elicit smaller N400 when they are preceded by associated words than when they are preceded by unassociated words (Bentin, 1987). Likewise, when words appear in a congruous sentence context, they elicit smaller N400 than when they appear in an incongruous context (Kutas \& Hillyard, 1980). The N400 elicited by words is typically largest at centroparietal electrode sites and is slightly larger over RH than it is over LH scalp sites (Kutas, Van Petten, \& Besson, 1988). However, functional magnetic resonance imaging (Kuperberg et al., 2000) and magnetoencephalography (Halgren et al., 2002) studies suggest that the N400 is generated by bilateral sources, with a LH bias (see also Hagoort, Brown, \& Swaab, 1996; Kutas, Hillyard, \& Gazzaniga, 1988).

The paradoxical distribution of the N400 component demonstrates the difficulty in inferring the neural generators of ERP components on the basis of their location on the scalp (Dale \& Sereno, 1993). The topography of ERP components such as the 
N1, generated (for the most part) by a small set of neural sources near the lateral surface of the brain, bears a relatively straightforward relationship to its neural generators (Hopf, Vogel, Woodman, Heize, \& Luck, 2002). However, because the N400 is generated by a large set of broadly distributed sources, the relationship between the location of the neural generators and the topography of the component at the scalp is more complex (Halgren et al., 2002; Van Petten \& Rheinfelder, 1995). Nonetheless, changes in the topography of an ERP component as a function of an experimental manipulation do indicate the operation of a slightly different set of neural generators in the respective conditions-although the exact configuration of these generators cannot be inferred without evidence from other neuroimaging techniques (Urbach \& Kutas, 2002).

\section{The Present Study}

In this study, we used ERPs to examine the response to lateralized target words that were preceded by lexically associated or unassociated primes. The same word pairs were used in two experiments, occurring out of context (in list format) in Experiment 1 and in sentences in Experiment 2 (see Table 2 for sample stimuli). Associated word pairs were embedded in sentences in which they either made sense together (congruous associated) or did not (incongruous associated). Word and sentence-level context were fully crossed via the inclusion of congruous sentences without associates (congruous unassociated) and incongruous sentences without associates (incongruous unassociated). It is important to note that the cloze probabilities of both sorts of congruous endings (associated and unassociated) were matched, as were those of the associated and unassociated incongruous endings. Matching for cloze probability ensures that message-level constraints are similar in the associated and unassociated conditions, so that the critical factor is the presence or absence of a lexical associate of the target words.

Primes (word as well as sentence) were presented centrally so that contextual information was potentially available to both hemispheres. ERPs to lateralized target words eventually reflect the whole brain's response to these stimuli, though hemifield presentation is assumed to shift the balance of processing of the critical stimuli toward the contralateral hemisphere. As in behavioral studies, in our analyses, differences in hemispheric sensitivity to experimental factors are inferred from the nature of their interactions with visual field of presentation on the amplitude and/or latency of various ERP components. Further, the degree to which hemifield presentation successfully shifts the balance of processing to the contralateral hemisphere is indexed by changes in the topography of ERPs as a function of VF of presentation (and evidenced in our analyses by interactions between VF and scalp topography factors such as hemisphere, laterality, and anteriority). We evaluated the sensitivity of each hemisphere's processing of word- versus sentence-level context by measuring the impact of $\mathrm{VF}$ on the size of N400 context effects. For example, as both hemispheres have previously been shown to be sensitive to wordlevel context, in Experiment 1, we predicted similar-sized N400 context effects (less negative N400 for associated than for unassociated targets) with presentation to both the LVF and the RVF.

In Experiment 2, however, N400 effects might be expected to differ as a function of VF, with RVF presentation yielding greater
N400 sentence congruity effects. Because prior research in the hemifield presentation paradigm indicates $\mathrm{LH}$ sensitivity to message-level context, we predicted that RVF presentation would yield ERP effects similar to those observed with central presentation. Research with central presentation has suggested that the impact of lexical association is attenuated by increasing amounts of sentence-level contextual information (Kutas, 1993; Van Petten, 1993) and, indeed, can be overridden by the strong sentence contexts used in Experiment 2 (Van Petten et al., 1999). Thus, with RVF presentation, we might expect to observe a large N400 congruity effect (less negative N400 to congruous sentence completions) and negligible effects of association. Moreover, the message-blind RH model, which asserts that the RH is sensitive only to word-level context, predicts that LVF presentation will yield a large N400 association effect (less negative N400 to associated than to unassociated words) and negligible effects of congruity.

\section{Experiment 1}

One of the most basic findings in psycholinguistics is the semantic priming effect: Response latencies to a word are reduced when it is preceded by a semantically or associatively related word (Meyer \& Schvaneveldt, 1971). ERP measures are also sensitive to these relationships (see Osterhout \& Holcomb, 1995, for review). In particular, the N400 to a target word is smaller when it is preceded by a related or associated word than when it is preceded by an unrelated word (Bentin, 1987; Holcomb, 1988). It is presumable that primed words elicit smaller N400s than unprimed words because they are easier to process. As a consequence, the difference between the amplitudes of the N400 elicited by primed and unprimed words can be taken as an electrophysiological index of semantic priming. The presence or absence of the N400 priming effect following laterally presented words thus can reflect each hemisphere's sensitivity to word-level context.

In Experiment 1, we examined ERPs to the lateralized presentation of words preceded by either a related (lexically associated) or an unrelated (lexically unassociated) word. Work using the hemifield priming paradigm with naming and lexical decision tasks has demonstrated associative priming with both LVF and RVF presentation; we thus expected similar patterns in the ERPs, with N400 amplitude reductions for associated relative to unassociated targets for both visual fields. One goal of Experiment 1 was to ensure that the critical words from Experiment 2 would elicit N400 word-level priming effects when presented laterally and to compare the size and timing of ERP associative priming effects as a function of visual field of presentation. The results of the experiment also establish an out-of-context baseline for comparison with the in-context priming effects examined in Experiment 2.

\section{Method}

\section{Materials}

Stimuli consisted of 160 pairs of associated words, such as spare and tire, and 160 unassociated counterparts, such as spare and pencil. The associated pairs were drawn from a published word production norm, the Edinburgh Associative Thesaurus (EAT, Coltheart, 1981; Kiss, Armstrong, Milroy, \& Piper, 1973). For these associated pairs, the second word was offered as the response to the first word by an average of 31 of the 100 
participants in the Edinburgh normative study (i.e., when presented with spare, $31 \%$ of the participants offered tire as a response; range $10 \%-85 \%$ across our set of associated pairs). It is noteworthy that across the more than 8,000 stimulus words included in the EAT, the average frequency for the most common response was $24 \%$, so that a mean frequency of $31 \%$ indicates a fairly strong associative relationship in the present stimulus set. Associated and unassociated target (second) words were matched for length (associated, 5.5 characters, $S E=0.1$; unassociated, 5.7 characters, $S E=0.1)$ and word frequency measured as the sum of all regularly inflected forms (Francis \& Kučera, 1982; associated, $M=180, S E=16$; unassociated, $M=194, S E=18$ ).

\section{Participants}

Participants were 16 healthy adults ( 8 women and 8 men), average age 20.2 years $(S D=2.8)$, who participated as part of a University of California, San Diego psychology or cognitive science course requirement. Participants all had normal or corrected-to-normal vision, and none reported any history of neurological or psychiatric disorders. All were right-handed, and none had left-handers in their immediate family.

\section{Procedure}

Participants were seated in a sound-attenuated, electrically shielded booth, approximately $40 \mathrm{in}$. from the computer monitor. Words were presented in black Helvetica font on a white background to maximize contrast and to facilitate the task of reading laterally presented target words. The second word of each pair appeared with a period (e.g., whole.), as it would in Experiment 2. Participants saw each prime and target only once and, across participants, all stimuli appeared equally in both VF conditions.

Each trial began with the presentation of a fixation cross for a duration that varied randomly from 1,000 to $1,200 \mathrm{~ms}$. The offset of the fixation cross was followed by the first word of a pair for $200 \mathrm{~ms}$ and then by a 300 -ms interstimulus interval. The second (target) word was then presented for $200 \mathrm{~ms}$ parafoveally such that its inside edge (the first character of a word in the RVF and the last character of a word in the LVF) was two degrees of horizontal visual angle from the fixation point. We ensured adequate perception by requiring participants to name sentence-final words: $2,500 \mathrm{~ms}$ after the offset of the target word, a blue question mark appeared in the center of the monitor, which cued the participant to name the laterally presented word, or to say "didn't see" if they were unable to read it. The next trial began $10 \mathrm{~s}$ after the onset of the naming prompt. The somewhat long intertrial interval was necessary in order to allow the bio-amplifiers to settle after the high amplitude electrical activity associated with the production of a spoken response.

Lateralization of the stimuli was assured by several aspects of the experimental procedure: (a) random mixing of right and left presentations, (b) brief presentations of the target words, and (c) monitoring of horizontal eye movements (via the electrooculogram as described in the next section), so that trials in which eye movements did occur could be rejected.

\section{ERP Recording and Data Analysis}

The electroencephalogram (EEG) was recorded with a commercially available electrode cap from Electrocap International (Eaton, Ohio) fitted with 26 tin electrodes arranged geodesically (to offer full coverage of the head). All electrodes were referenced to the left mastoid online and rereferenced offline to the average of activity at left and right mastoids. Blinks were monitored via electrodes placed on the lower orbital ridges, referenced to the left mastoid. Horizontal eye movements were monitored via a bipolar montage of electrodes placed at the outer canthi (the electrooculogram; EOG). Electrode impedances were kept below $5 \mathrm{k} \Omega$. The EEG and EOG were amplified by Grass amplifiers (Astro-Med, West
Warwick, Rhode Island) with a half-amplitude cutoff bandpass from 0.01 to $100 \mathrm{~Hz}$. The sampling rate was $250 \mathrm{~Hz}$.

ERPs were averaged offline for an epoch of 1,024 ms, beginning $100 \mathrm{~ms}$ before the onset of the target words. We rejected trials contaminated by eye artifacts or amplifier blocking (about $14.3 \%, S D=10.8$ ) prior to averaging. We calculated averages of artifact-free ERP trials for each type of target word in each hemifield after subtraction of the 100-ms prestimulus baseline. Only words that were correctly named in the delayed task were allowed to contribute to the ERP averages. Unless noted otherwise, data analysis involved repeated measures analysis of variance (ANOVA) with factors visual field (LVF or RVF), association (associated or unassociated), and three factors that index scalp topography: hemisphere (left or right), laterality (lateral or medial), and anterior-posterior (four levels from the front of the head to the back). VF always refers to the location of the stimulus, and hemisphere always refers to electrode site (over the left side of the scalp or the right). We conducted analyses for the 22 lateral scalp sites; 4 sites on the midline of the head are included in the figures but were not subjected to statistical analyses. In all analyses, $p$ values are reported after epsilon correction (Huynh-Feldt) for repeated measures with greater than one degree of freedom in the numerator.

\section{Results}

\section{Naming Accuracy}

Accuracy in the delayed naming task was higher for RVF than it was for LVF presentations, $F(1,15)=24.5, p<.001$, and for associated relative to unassociated words, $F(1,15)=17.34, p<$ .001. These factors also interacted, $F(1,15)=7.2, p<.05$, reflecting a larger association effect with $\mathrm{LVF} / \mathrm{rh}$ presentation (see Table 1). The association effect was reliable for stimuli presented to both VFs, LVF: $F(1,15)=13.2, p<.01$; RVF: $F(1,15)=$ $5.45, p<.05$.

\section{ERPs}

We analyzed artifact-free ERP responses to correctly named target words in four time windows, encompassing the N1 component (50-150 ms after stimulus onset), the P2 component (150$250 \mathrm{~ms}$ ), the $\mathrm{N} 400$ component (300-500 ms), and the late positive complex (500-900 ms).

One possible concern associated with the experimental paradigm employed in the present study is that, because of differences in naming accuracy in the left and right VFs, more LVF than RVF trials were eliminated from the ERP averages. The concern, of course, is that a disparity in the number of trials might result in a lower signal to noise ratio for LVF ERPs, thus resulting in a loss of statistical power. In fact, the average number of LVF trials (associated $=31.5$, unassociated $=27.4$ ) was fairly comparable to

Table 1

Naming Accuracy in Experiment 1

\begin{tabular}{lccccr}
\hline & \multicolumn{2}{c}{$\mathrm{RVF} / \mathrm{lh}$} & & \multicolumn{2}{c}{$\mathrm{LVF} / \mathrm{rh}$} \\
\cline { 2 - 3 } \cline { 5 - 6 } Word pair & $M$ & & & $M$ & $S D$ \\
\hline Associated & 97 & 4 & & 91 & 6 \\
Unassociated & 94 & 6 & & 78 & 15 \\
\hline
\end{tabular}

Note. $\mathrm{RVF} / \mathrm{lh}=$ right visual field presentation; $\mathrm{LVF} / \mathrm{rh}=$ left visual field presentation. 


\section{Left Occipital Site}

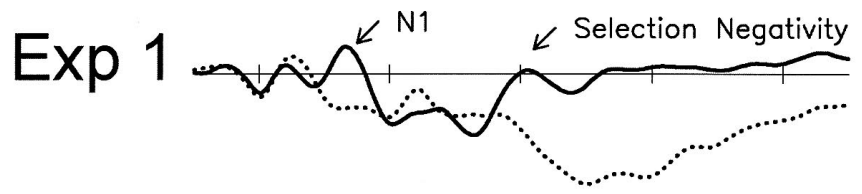

Exp 2

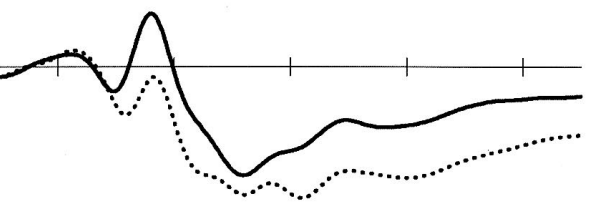

Right Visual Field

Left Visual Field

\section{Right Occipital Site}
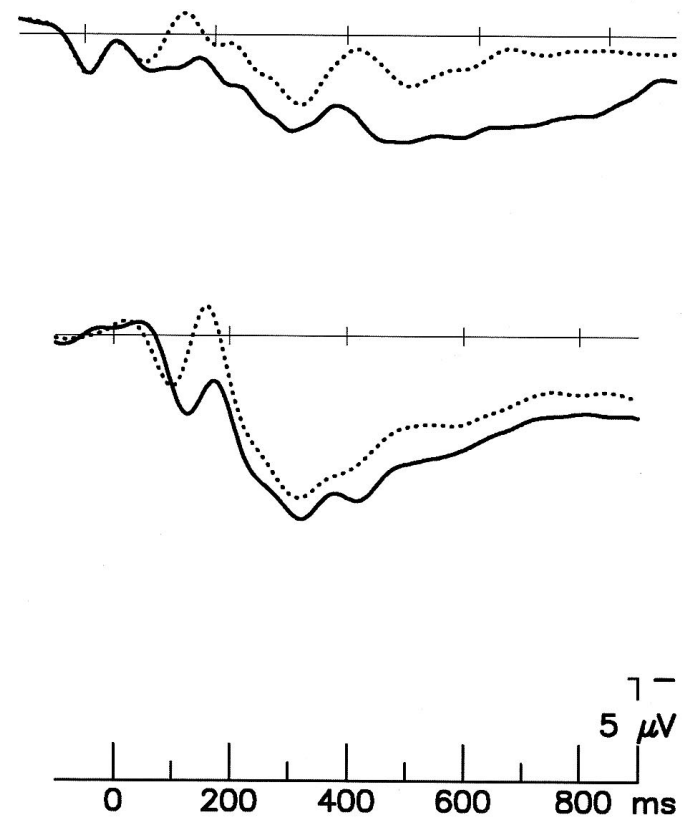

Figure 1. Grand average event-related brain potentials to stimuli presented to the right visual field and the left visual field in Experiments 1 and 2. The traces show data recorded from left and right occipital electrode sites where the N1 component is prominent. Negative voltage is plotted up. Note that the N1 is largest over the hemisphere contralateral to the visual field of presentation.

the average number of RVF trials (associated $=33.2$, unassociated $=33.5$ ). Because the signal-to-noise ratio changes as a function of the square of the number of trials, these small differences in the absolute number of trials are unlikely to substantively affect the noise level in the resultant ERPs. ${ }^{1}$

N1 $(50-150 \mathrm{~ms})$. To assess whether the lateralized presentation of the target words was effective in stimulating the contralateral hemisphere, one can examine visual potentials such as the N1. The N1 is presumed to reflect extrastriate visual processing and is largest contralateral to the VF of stimulation (Hillyard \& AnlloVento, 1998). Mean amplitude N1 responses (50-150 ms) showed a reliable VF $\times$ Hemisphere interaction, $F(1,15)=6.13, p<.05$. Under normal viewing conditions, the $\mathrm{N} 1$ to words is slightly larger over LH recording sites (King, Ganis, \& Kutas, 1998). With RVF presentation, this asymmetry was exacerbated $(\mathrm{LH}=-0.1$ $\mu \mathrm{v}, \mathrm{RH}=0.6 \mu \mathrm{v})$; with LVF presentation, this asymmetry was reduced $(\mathrm{LH}=0.6 \mu \mathrm{v}, \mathrm{RH}=0.5 \mu \mathrm{v})$. The N1 was larger (less positive) over scalp sites contralateral to the stimulated VF (see Figure 1). There was no effect of word association in the N1 latency window.

$P 2(150-250 \mathrm{~ms})$. The N1 response is typically followed by a positive peak, the $\mathrm{P} 2$, which has been linked to high-level visual processing, including target detection in visual search paradigms (Luck \& Hillyard, 1994). P2 amplitudes also have also been found to vary as a function of psycholinguistic variables and VF of presentation (Federmeier \& Kutas, 2002). Figures 2 and 3 show ERPs elicited by associated and unassociated words in the two visual fields. Mean amplitude $\mathrm{P} 2$ responses were generally more positive for RVF than for the LVF presentations, main effect of VF: $F(1,15)=13.2, p<.01$. The impact of visual field varied across the scalp, as indicated by interactions between VF and the scalp location factors, $\mathrm{VF} \times$ Hemisphere, $\mathrm{VF} \times$ Laterality, $\mathrm{VF} \times$ Hemisphere $\times$ Laterality; all $F \mathrm{~s}(1,15)>7.65$, all $p \mathrm{~s}<.05$. The impact of presentation field was particularly pronounced over right lateral scalp sites in this time window, continuing the pattern observed in the $\mathrm{N} 1$ time window. In contrast, responses over left-scalp sites did not differ as a function of VF.

There was also a main effect of association on P2 amplitudes, $F(1,15)=25.5, p<.001$, that was especially evident over medial electrode sites, Association $\times$ Laterality: $F(1,15)=5.16, p<.05$. ERPs were more positive to associated than to unassociated targets. However, there was no Association $\times$ VF interaction.

\footnotetext{
${ }^{1}$ To confirm this, we measured the root-mean-square (RMS) amplitude in the prestimulus portion ( $-100-0 \mathrm{~ms})$ of ERPs elicited by LVF and RVF stimuli. A measure of signal power, the RMS is calculated by taking the square root of the mean sum of squares over the interval. Whereas voltage fluctuations above and below zero cancel out in mean amplitude measurements, they do not do so in measures of RMS. RMS thus serves as a liberal estimate in the noise (construed as nonstimulus locked activity) in the ERP. Analysis of these values revealed a null effect of VF $(F<1)$ and no significant interactions between $\mathrm{VF}$ and any of the topography factors (hemisphere, laterality, and anterior-posterior), suggesting that the noise level was similar in LVF and RVF ERPs.
} 


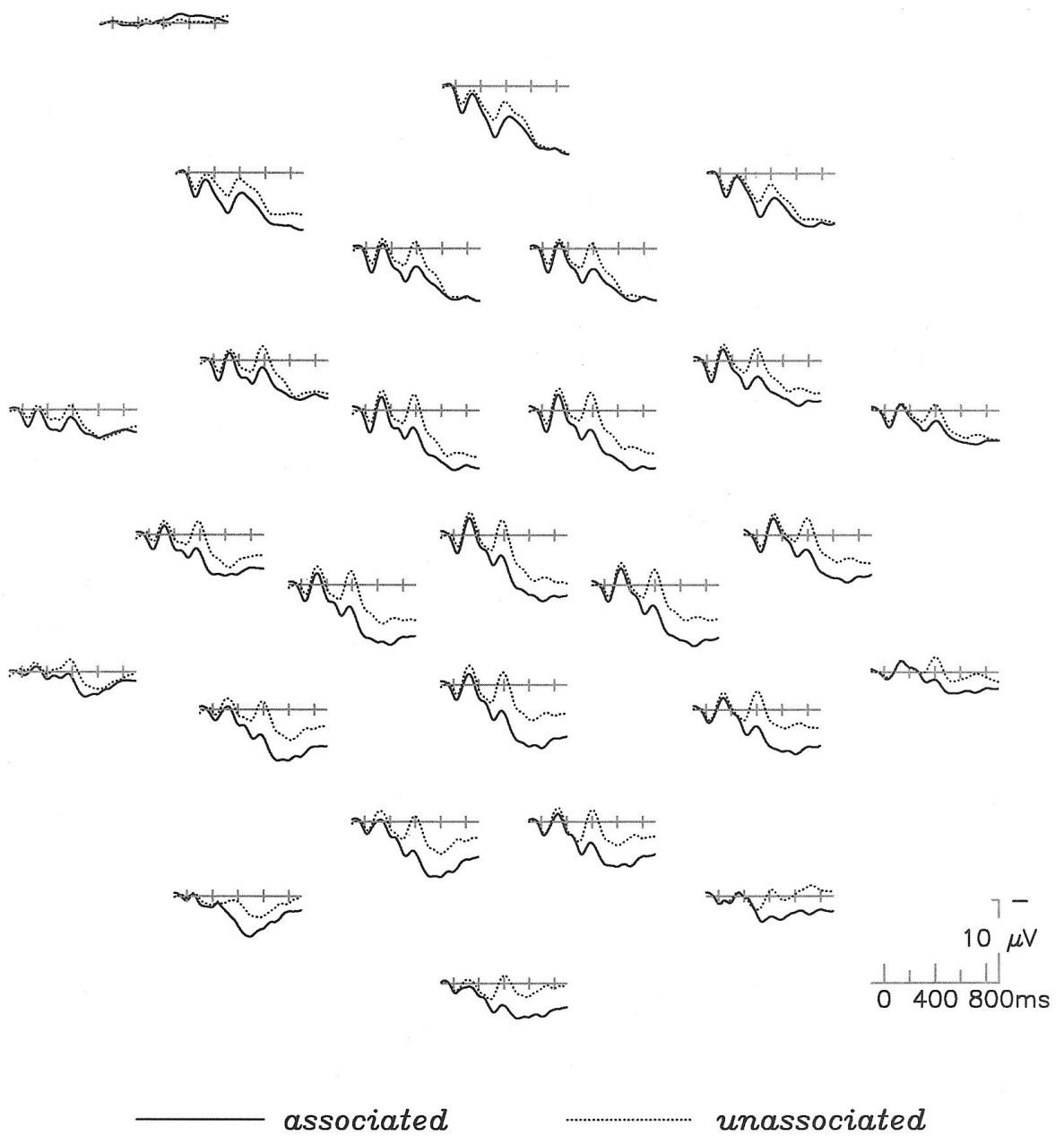

Figure 2. Grand average event-related brain potentials to correctly named words in the left visual field in Experiment 1. Layout of electrode sites in the figure approximates their spatial location on the scalp, with midline prefrontal top and center and right occipital bottom right. Negative voltage is plotted up. The trace in the upper left corner is the electroocculogram, a bipolar montage of the electrical signal recorded at the side of the left eye, referenced to the side of the right eye.

N400 (300-500 ms). Analysis of the N400 measures yielded a main effect of VF, $F(1,15)=6.04, p<.05$, and interactions between $\mathrm{VF}$ and all of the topographic variables, $\mathrm{VF} \times \mathrm{Hemi-}$ sphere: $F(1,15)=14.3, p<.01 ; \mathrm{VF} \times$ Hemisphere $\times$ Laterality: $F(1,15)=10.7, p<.01 ; \mathrm{VF} \times$ Hemisphere $\times$ Anterior-Posterior: $F(3,45)=14.8, p<.01$. This pattern of interaction results because of a contralateral selection negativity over lateral, posterior electrode sites. Stimuli presented to the LVF elicited more negative ERPs over right lateral posterior sites, and stimuli presented to the RVF elicited a mirror image pattern of responses. This response pattern has been observed in several prior studies using lateralized stimuli (Federmeier \& Kutas, 1999, 2002; Neville, Kutas, \& Schmidt, 1982).

There was also a main effect of association; Figures 2 and 3 show that unassociated words elicited larger N400s than associated words, $F(1,15)=44.6, p<.0001$. This effect was largest over medial central-posterior sites and was slightly larger over the right, Association $\times$ Laterality: $F(1,15)=30.0, p<.001$;
Association $\times$ Hemisphere $\times$ Laterality: $F(1,15)=5.10, p<.05$; Association $\times$ Laterality $\times$ Anterior-Posterior: $F(3,45)=8.66$, $p<.001$; Association $\times$ Hemisphere $\times$ Laterality $\times$ Anterior Posterior: $F(3,45)=2.87, p<.05$. This is the distribution typically observed for N400 responses to visual words (e.g., Kutas et al., 2000; Kutas \& Van Petten, 1994). The association effect was slightly larger with RVF presentation over medial sites where N400 effects were more prominent, $\mathrm{VF} \times$ Association $\times$ Laterality: $F(1,15)=5.26, p<.05$.

We followed up on this interaction using analyses performed within each VF condition separately. With RVF presentation, there was a reliable $3.6 \mu \mathrm{V}$ effect of association, $F(1,15)=29.0, p<$ .001 ; this effect was most pronounced over medial, centralposterior electrode sites, Association $\times$ Laterality: $F(1,15)=$ 16.0, $p<.01$; Association $\times$ Laterality $\times$ Anterior-Posterior: $F(3$, $45)=5.64, p<.01$. With LVF presentations, the effect of association was again reliable but was $3 \mu \mathrm{V}$ in size, $F(1,15)=$ $32.8, p<.0001$. This effect was also largest over medial central- 


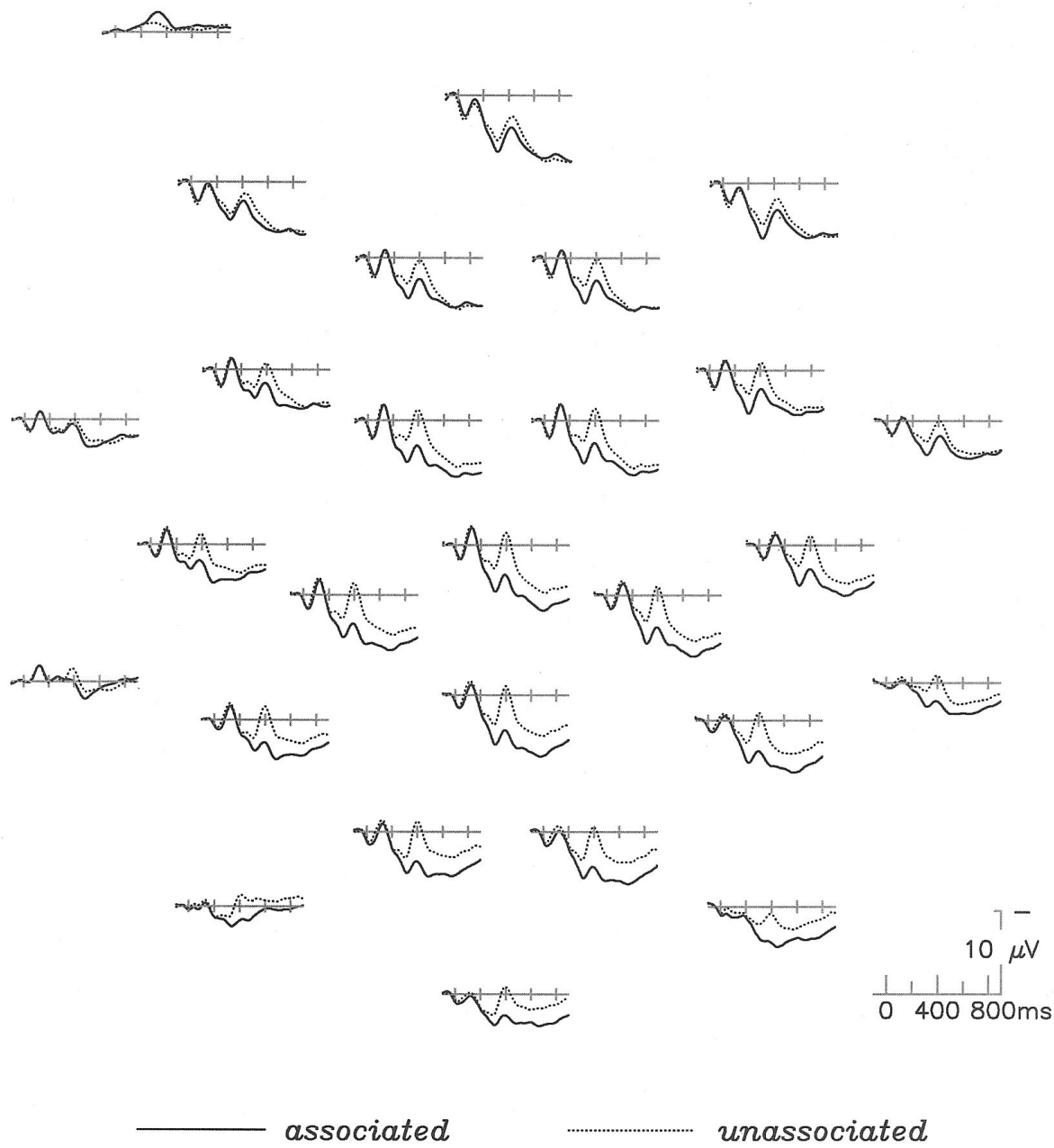

Figure 3. Grand average event-related brain potentials to correctly named words in the right visual field in Experiment 1. Layout of electrode sites in the figure approximates their spatial location on the scalp, with midline prefrontal top and center and right occipital bottom right. Negative voltage is plotted up. The trace in the upper left corner is the electroocculogram, a bipolar montage of the electrical signal recorded at the side of the left eye, referenced to the side of the right eye.

posterior electrode sites, with a greater right lateralization than was observed with RVF presentation, Association $\times$ Laterality: $F(1$, $15)=32.3, p<.0001$; Association $\times$ Hemisphere $\times$ Laterality: $F(1,15)=9.35, p<.01$; Association $\times$ Laterality $\times$ AnteriorPosterior: $F(3,45)=4.95, p<.05$; Association $\times$ Hemisphere $\times$ Laterality $\times$ Anterior-Posterior: $F(3,45)=3.52, p<.05$.

We assessed the timing of the N400 association effect in each VF by measuring difference waves formed by point-by-point subtraction of the amplitude of ERPs to associated stimuli from those to unassociated stimuli presented in the same visual field. Because the difference wave represents the association effect in each visual field, one can assess the timing of this effect by measuring the latency of the onset and the peak of these waveforms. We measured peak latencies of the LVF and RVF association difference waves and subjected them to repeated measures ANOVAs with factors visual field (LVF or RVF) and the same scalp topography factors employed in the analyses above (hemisphere, laterality, and anteriority). The N400 association effect peaked at $404 \mathrm{~ms}$ after word onset with LVF presentation and at $402 \mathrm{~ms}$ postonset with RVF presentation. The main effect of VF was not significant $(F<$ 1 ), and neither were any of the interactions between VF and topographic factors (all two- and three-way interactions, $F \mathrm{~s}<1$; four-way interaction, $F=2.15, n s$ ).

We measured the onset of the N400 association effect by determining the latency at which the difference wave reached $10 \%$ of its peak amplitude. We subjected these values to an analysis similar to that used for the peak latencies. The main effect of VF was not significant $(F<1)$, though analysis did reveal a reliable $\mathrm{VF} \times$ Laterality $\times$ Anteriority interaction, $F(3,45)=4.68, p<$ .01. The latter resulted because the onset of effects at anterior sites was earlier with LVF presentation, whereas the onset of effects at posterior sites was earlier with RVF presentation. None of the other interactions between VF and topographic factors was reliable $\left(F_{\mathrm{s}} \leq 1.97\right)$

Late positive complex (500-900 ms). Although the 300-500 ms latency range encompasses the peak of the N400, Figures 2 and 
3 show that the impact of lexical association continued to $900 \mathrm{~ms}$ after stimulus onset at some scalp sites. The late portion of the association effect can be considered the late phase of the N400 but may also receive contributions from other processes, so that the 500-900 ms epoch was analyzed separately. In this latency range, there was no main effect of VF, but interactions between VF and topographic factors were observed because of the continuation of the selection negativity, VF $\times$ Hemisphere: $F(1,15)=24.1, p<$ $.001 ; \mathrm{VF} \times$ Hemisphere $\times$ Laterality: $F(1,15)=25.7, p<.001$; $\mathrm{VF} \times$ Hemisphere $\times$ Anterior-Posterior: $F(3,45)=65.0, p<$ .0001 ; VF $\times$ Hemisphere $\times$ Laterality $\times$ Anterior-Posterior: $F(3$, $45)=25.3, p<.0001$.

Continuing the pattern seen in the N400 time window, there was a main effect of association, with more positive responses to associated than to unassociated words, $F(1,15)=28.2, p<.001$. The difference in this time window was particularly pronounced at medial posterior electrode sites over the right hemisphere, Association $\times$ Laterality: $F(1,15)=11.3, p<.01$; Association $\times$ Hemisphere $\times$ Anterior-Posterior: $F(3,45)=3.41, p<.05$; Association $\times$ Laterality $\times$ Anterior-Posterior: $F(3,45)=11.5$, $p<.0001$; Association $\times$ Hemisphere $\times$ Laterality $\times$ AnteriorPosterior: $F(3,45)=9.79, p<.0001$.

VF and association did not interact in this analysis either when considered alone or as a function of topographic variables. However, visual inspection suggested a possible VF effect around 700 $\mathrm{ms}$ after word onset. We therefore conducted a post hoc analysis on the difference waves (created by taking a point-by-point subtraction of the unassociated minus associated waveform for each VF condition), measuring the mean amplitude difference between 600 and $800 \mathrm{~ms}$ in each VF. This analysis suggested that over the right hemisphere, especially at lateral electrode sites, the association effect (increased positivity to associated as compared with unassociated items) in this interval was indeed larger with LVF presentation, VF $\times$ Hemisphere $\times$ Laterality: $F(3,45)=3.93$, $p<.05$.

Summary. Lateralized presentation of the stimuli resulted in asymmetric $\mathrm{N} 1$ responses and a temporally extended contralateral selection negativity over posterior electrodes (Figure 1). For presentation in both VFs (Figures 2 and 3), associated words elicited more positive responses beginning with the $\mathrm{P} 2$ and continuing through the N400 time window into the LPC time window (i.e., from 150 to $900 \mathrm{~ms}$ ). Association effects were larger for RVF/lh than for LVF/rh presentations during the peak N400 latency window, but the onset and the peak of this effect were similar. Following the peak of the N400, the association effect was somewhat larger for LVF/rh presentations 600-800 ms postonset.

\section{Discussion}

Although performance on the delayed naming task was quite good, responses were more accurate with $\mathrm{RVF} / \mathrm{lh}$ presentation, confirming the well-known left-hemisphere superiority for naming (Gazzaniga \& Sperry, 1967). Responses to associated targets were reliably more accurate than responses to unassociated targets with presentation to either VF. Results of the delayed naming task thus indicate that the word pairs employed in the present study engender associative priming. Although the association effect was greater with presentation to the $\mathrm{LVF} / \mathrm{rh}$ than with presentation to the $\mathrm{RVF} / \mathrm{lh}$, the interaction may be less a reflection of greater right-hemisphere sensitivity to word-level priming than a function of the near-ceiling performance with $\mathrm{RVF} / \mathrm{lh}$ presentation (see Table 1).

Associative priming was also evident in the ERPs. With presentation to both the RVF and the LVF, targets preceded by a lexical associate elicited more positive ERPs than did the unassociated targets. Association effects were first evident 150-250 ms after the onset of the targets in the interval of the P2 component. Though the functional significance of this ERP component is not fully understood, the P2 is thought in part to index high-level visual processing. Enhanced P2 to associated targets thus may reflect enhanced visual processing of these stimuli relative to the unassociated targets. In any case, the similar-sized association effect in both VFs revealed no indication of hemispheric asymmetry in this aspect of processing.

Association effects were also evident on the N400, as ERPs were less negative (more positive) for associated than for unassociated targets. This replicates prior findings with centrally presented stimuli of reduced N400 amplitudes with lexical association, indicating decreased processing difficulty for words preceded by an associate (Bentin, 1987; Holcomb, 1988). In the present study, the N400 priming effect was slightly larger with presentation to the RVF, suggesting that the left hemisphere was more able to capitalize on the semantic relationships in the associated word pairs. These effects observed on the N400 component continued into the latter part of the epoch, with greater positivity between 500 and $900 \mathrm{~ms}$ after stimulus onset for associated than for unassociated targets. During the entire LPC interval, the size of this effect was the same with RVF and LVF presentation. However, (post hoc) measurements restricted to $600-800 \mathrm{~ms}$ after target onset revealed a somewhat larger priming effect with LVF (right hemisphere) presentation.

Our finding of robust associative priming effects with presentation to both the RVF and the LVF mirrors that of the behavioral literature, in which lexical priming is typically observed in both VFs under similar experimental conditions, in other words, centrally presented primes and a stimulus onset asynchrony (SOA) of 500 ms (Chiarello, 1998; Chiarello, Liu, Shears, Quan, \& Kacinik, 2003). In the present study, lexical associates were chosen on the basis of their congruency in the sentence materials employed in Experiment 2. As a consequence, they included pairs with a mix of types of relatedness, including some that shared category membership, some related idiomatically, and some associated through other kinds of linking schemas. As such, these data cannot speak to the issue of whether categorical relationships are processed similarly in the two cerebral hemispheres (c.f. Chiarello, 2000; Koivisto \& Laine, 2000). However, results of the present study are consistent with hemifield studies of stimuli chosen purely on the basis of associative strength. Coney (2002), for example, reported a linear relationship between associative strength and lexical decision times in both visual fields.

Previous researchers have addressed the temporal availability of word meanings by varying the SOA between the prime and the target. In such studies, short SOAs (fewer than $300 \mathrm{~ms}$ ) often yield priming effects only with presentation to the RVF/lh and not to the $\mathrm{LVF} / \mathrm{rh}$, whereas longer SOAs (over 1,000 ms) yield the opposite pattern, with priming effects for only $\mathrm{LVF} / \mathrm{rh}$ and not $\mathrm{RVF} / \mathrm{lh}$ presentation (e.g., Burgess \& Simpson, 1988). Observations such as these have led to the suggestion that lexical activation in the 
right hemisphere begins later and lasts longer than in the left hemisphere. One might expect, then, that the delayed onset and protracted duration of RH lexical activation might be reflected in this study by the onset and offset of ERP effects, as the ERP provides a continuous and temporally precise measure of processing.

In fact, regardless of $\mathrm{VF}$, priming effects began in the interval $150-250 \mathrm{~ms}$ after the onset of the target. Although VF of presentation affected the size of the priming effect, it did not seem to affect the overall character of the ERP response. Both LVF and RVF presentation resulted in enhanced P2 for associated stimuli relative to unassociated stimuli and a reduction in N400 amplitude that extended into the time window of the late positive complex. These results thus do not support a notable hemispheric asymmetry in the onset of lexical activation - at least in the processing of a target stimulus presented $500 \mathrm{~ms}$ after the onset of the prime.

Differences in the size of the priming effect as a function of $\mathrm{VF}$, however, might support a more subtle asymmetry in the timecourse of associative priming. As noted above, ERP priming effects were larger with RVF presentation 300-500 ms after stimulus onset but larger with LVF presentation 600-800 ms after stimulus onset. So, although LVF and RVF ERP priming effects began at the same time, the RVF/lh effects were larger earlier in the epoch, whereas LVF/rh effects were larger later on. Perhaps this pattern of effects reflects hemispheric differences in the degree of activation, with more early activation in the left hemisphere and more late activation in the right hemisphere. The present findings suggest, however, hemispheric differences in the degree of semantic activation over time rather than any abrupt difference in activation onsets or offsets.

\section{Experiment 2}

Experiment 1 revealed that unassociated word pairs such as spare pencil elicit larger N400 responses than do associated pairs such as spare tire in both visual fields. In Experiment 2, ERPs were recorded as participants read these word pairs in sentence contexts. The stimuli consisted of 160 quartets of sentences formed by crossing two factors: the plausibility of the final word as a sentence completion (congruous or incongruous) and the occurrence of a lexical associate of the final word earlier in the sentence (associated or unassociated). Examples of the sentence stimuli are shown in Table 2.

When centrally presented in a previous study, ERPs to the final words of these sentences showed a robust effect of sentence congruity, consisting largely of smaller N400s for congruous endings. No impact of lexical association on N400 amplitude was observed (Van Petten et al., 1999). This pattern of results stands in contrast with other work showing that lexical associates embedded in sentence-like but meaningless word strings (After fixing the movie she found they should have killed left instead of right at the pot.) do lead to reduced N400s for the second words of the associated pairs (Schwartz, Federmeier, Van Petten, Salmon, \& Kutas, 2003; Van Petten, 1993; Van Petten, Weckerly, McIsaac, \& Kutas, 1997). The contrasting results indicate that both lexical association and sentence congruity can exert independent influences on N400 amplitude in word strings but that when these influences are opposed, sentence-level context tends to override lexical association.
Table 2

Sample Stimuli in Experiment 2

\section{Congruous associated}

He was pretty confused after the attack, but the story came out in bits and PIECES.

They were truly stuck, since she didn't have a spare TIRE.

The Italian cook always added too much olive OIL.

There are some kids who will only eat macaroni with CHEESE.

\section{Congruous unassociated}

They advertise it as a nutritious granola bar, but it's also full of bits of CHOCOLATE.

During the test, Ellen leaned over and borrowed my spare PENCIL.

They were hard to walk in, but she loved her olive SHOES.

To make his art project, the kid stuck on the macaroni with GLUE.

\section{Incongruous associated}

They advertise it as a nutritious granola bar, but it's also full of bits of PIECES.

During the test, Ellen leaned over and borrowed my spare TIRE.

They were hard to walk in, but she loved her olive OIL.

To make his art project, the kid stuck on the macaroni with CHEESE.

\section{Incongruous unassociated}

He was pretty confused after the attack, but the story came out in bits and CHOCOLATE.

They were truly stuck, since she didn't have a spare PENCIL.

The Italian cook always added too much olive SHOES.

There are some kids who will only eat macaroni with GLUE.

Note. Sentence-intermediate lexical associates are shown in italics, and final words appear in capitals only for illustration. During the experiment, all sentence words were presented in standard font with capitalization as appropriate.

In Experiment 2, sentence contexts were presented centrally, so that both hemispheres could contribute normally to the formation of discourse-level representations that support sentence congruity effects. By increasing the participation of the contralateral hemisphere, we intended lateralized presentation of sentence-final words to gauge the relative influence of message-level congruity and word-level associative information on the real-time processing of words by each hemisphere. Because N400 amplitude is sensitive to both word and sentence-level context effects, it served as our main dependent variable.

Because the LH is known to be critical for sentence-level processing, we expected RVF presentation to yield a pattern of results much like that of central presentation: large sentence congruity effects but negligible influences of lexical association. If the RH is also sensitive to message-level context, then we should also see an N400 congruity effect with LVF presentation, regardless of the presence of a lexical associate. By contrast, the message-blind $\mathrm{RH}$ model, in which the RH is primarily sensitive to word-level context, predicts an absence of sentence congruity effects with LVF presentation and similar N400 association effects (larger N400s for unassociated than for associated stimuli, as in Experiment 1 ) in the congruous and the incongruous sentence contexts.

\section{Method}

\section{Materials}

Cloze probabilities of the congruous sentence completions were established in a separate group of participants who participated for course credit 
in a University of California, San Diego introductory psychology course. Each participant received about 20 sentence frames without their final words and was asked to fill in the word she or he thought best completed the sentence. Each participant received an equal number of sentence frames corresponding to associated and unassociated sentences and saw only one version of each. Each of the sentence frames was completed by $66-76$ participants. Mean cloze probabilities for the final words in the congruous associated and congruous unassociated sentences were both $71 \%$. The incongruous final words were never offered as completions, so the cloze probability of these words was $0 \%$. The mean number of words in the associated sentences was $13.9(S E=0.3)$ and was $15.3(S E=0.3)$ in the unassociated sentences. Sentence-final words were the same as those used in Experiment 1.

The 160 quartets yielded 640 sentences total; each participant read half of these, 80 of each of the four sentence types. For each sentence type, half of the targets (40) appeared in participants' LVF and, and half appeared in the RVF, randomly intermixed. Stimuli were counterbalanced such that, for each quartet, a given participant saw either both of the congruous sentences or both of the incongruous sentences. Note that the two congruous (or two incongruous) sentences in a quartet share one intermediate word, but the sentence frames and final words are otherwise different. As a consequence, this counterbalancing scheme involves little within-subject stimulus repetition.

\section{Participants}

Participants were 20 healthy adults ( 10 women and 10 men), average age 21.65 years $(S D=4)$, who participated as part of a cognitive science or psychology course requirement. Data were recorded from 4 additional participants but were dropped from the analysis because of excessive artifacts in the EEG. Participants all had normal or corrected-to-normal vision and reported no history of neurological or psychiatric disorders. All participants were right-handed with no family history of sinistrality.

\section{Procedure}

As in Experiment 1, participants were seated in a sound-attenuated, electrically shielded booth approximately $40 \mathrm{in}$. from the computer monitor on which stimuli were presented. Sentences were presented one word at a time (two words per second) in black Helvetica font on a white background. Except for the sentence-final word, all words were presented centrally for $200 \mathrm{~ms}$ followed by $300 \mathrm{~ms}$ of blank screen. Sentence-final words were presented for $200 \mathrm{~ms}$ parafoveally, such that the leading edge (the first character of a word in the RVF, and the last character of a word in the LVF) subtended two degrees of horizontal visual angle from the fixation point.

Participants' task was to silently read sentences presented one word at a time in the center of the computer monitor and to name the sentence-final target word when cued by a prompt $2,500 \mathrm{~ms}$ after the target's offset. To ensure that participants fully processed the sentences, each trial was also followed by a yes-no comprehension probe that either accurately or inaccurately paraphrased the preceding sentence. As in Experiment 1, there were $10 \mathrm{~s}$ between the onset of the naming prompt and the beginning of the next trial; this interval was partially filled with the presentation of and response to the comprehension question.

\section{ERP Recording and Data Analysis}

Electrophysiological methods were as in Experiment 1. As in Experiment 1, data analysis was performed only for words that had been correctly named in the delayed naming task. Statistical analysis involved repeated measures ANOVAs conducted on the mean amplitude of ERP waveforms measured in several specific time intervals as described in the next section. Unless noted otherwise, factors in the analyses included VF (LVF or RVF), sentence congruity (congruous or incongruous), association (associated or unassociated), and the three scalp topography factors of hemisphere (left or right), laterality (lateral or medial), and anterior-posterior (four levels, from the front of the head to the back).

\section{Results}

\section{Naming Accuracy}

Naming accuracies are shown in Table 3. Overall, responses were more accurate for presentation to the RVF, $F(1,19)=58.8$, $p<.0001$, for congruous completions, $F(1,19)=64.0, p<.0001$, and for associated items, $F(1,19)=13.7, p<.01$. The main effects were qualified by $\mathrm{VF} \times$ Congruity interactions: $F(1,19)=$ 50.4, $p<.0001 ; \mathrm{VF} \times$ Association: $F(1,19)=41.3, p<.0001$; Congruity $\times$ Association: $F(1,19)=29.5, p<.0001$; and a three-way interaction among all variables: $F(1,19)=6.93$, $p<.05$.

Follow-up analyses showed that, for presentation to the LVF, naming was more accurate for congruous items, $F(1,19)=54.4$, $p<.0001$, and for those that were part of an associated pair, $F(1$, $19)=65.0, p<.0001$. However, there was a Congruity $\times$ Association interaction, $F(1,19)=25.5, p<.001$, with an improvement in naming accuracy for associated items only within the incongruous sentence contexts. For the RVF, naming accuracy was quite high overall, so that ceiling effects precluded main effects of either congruity or association. Accuracy was, however, lowest in the incongruent unassociated condition, leading to a Congruity $\times$ Association interaction, $F(1,19)=20.7, p<.001$.

\section{Sentence Comprehension Scores}

The comprehension questions were scored only when the final word had also been named correctly; accuracies are shown in Table 4. Accuracy was generally high $(M=92.4 \%, S D=6 \%)$, indicating that when participants were able to read the sentencefinal word, they typically understood the sentence as well. VF of presentation did not significantly modulate comprehension, $F(1$, $19)=2.99, p=.10$, and it did not interact with any other experimental variables (all $F \mathrm{~s}<1$ ). Participants scored higher on questions that followed congruous sentences than on questions that followed incongruous ones, $F(1,19)=25.2, p<.0001$. A Congruity $\times$ Association interaction, $F(1,19)=13.3, p<.01$, reflected a $3 \%$ advantage for congruous unassociated over congruous associated not seen in the analogous incongruous sentences.

Table 3

Naming Accuracy in Experiment 2

\begin{tabular}{|c|c|c|c|c|c|c|c|c|}
\hline \multirow[b]{3}{*}{ Word pair } & \multicolumn{4}{|c|}{ RVF } & \multicolumn{4}{|c|}{ LVF } \\
\hline & \multicolumn{2}{|c|}{ Congruous } & \multicolumn{2}{|c|}{ Incongruous } & \multicolumn{2}{|c|}{ Congruous } & \multicolumn{2}{|c|}{ Incongruous } \\
\hline & $M$ & $S D$ & $M$ & $S D$ & $M$ & $S D$ & $M$ & $S D$ \\
\hline Associated & 96 & 4 & 98 & 3 & 96 & 4 & 84 & 12 \\
\hline Unassociated & 99 & 1 & 94 & 5 & 95 & 4 & 69 & 16 \\
\hline
\end{tabular}

Note. $\quad \mathrm{RVF}=$ right visual hemifield $; \mathrm{LVF}=$ left visual hemifield. 
Table 4

Comprehension Accuracy in Experiment 2

\begin{tabular}{|c|c|c|c|c|c|c|c|c|}
\hline \multirow[b]{3}{*}{ Word pair } & \multicolumn{4}{|c|}{ RVF } & \multicolumn{4}{|c|}{ LVF } \\
\hline & \multicolumn{2}{|c|}{ Congruous } & \multicolumn{2}{|c|}{ Incongruous } & \multicolumn{2}{|c|}{ Congruous } & \multicolumn{2}{|c|}{ Incongruous } \\
\hline & $M$ & $S D$ & $M$ & $S D$ & $M$ & $S D$ & $M$ & $S D$ \\
\hline Associated & 92 & 6 & 90 & 6 & 93 & 5 & 92 & 6 \\
\hline Unassociated & 95 & 4 & 90 & 8 & 96 & 4 & 90 & 6 \\
\hline
\end{tabular}

Note. $\quad \mathrm{RVF}=$ right visual hemifield; $\mathrm{LVF}=$ left visual hemifield.

\section{ERPs}

As in Experiment 1, differences in naming accuracy in the two VFs could have led to differences in the noise level of the resultant LVF and RVF ERPs because of the inclusion of fewer trials in LVF ERPs. Though there were reliably fewer trials in LVF than in RVF ERPs, the actual disparities were rather small: congruous associated, 22.2 versus 23.7 trials; congruous unassociated, 21.2 versus 23.1 trials; incongruous associated, 20.2 versus 23.6 trials; and incongruous unassociated, 17.2 versus 24.6. These differences in the number of trials were determined to have negligible effects on the relative noise level. ${ }^{2}$

$\mathrm{N1}(50-150 \mathrm{~ms})$. As in Experiment 1, we subjected the mean amplitudes of the ERPs elicited between 50 and $150 \mathrm{~ms}$ after word onset to repeated measures ANOVAs with factors visual field (LVF or RVF) and the three scalp topography factors. This analysis revealed a reliable interaction between $\mathrm{VF}$ and all scalp topography factors, $\mathrm{VF} \times$ Hemisphere: $F(1,19)=10.19, p<.01$; $\mathrm{VF} \times$ Hemisphere $\times$ Laterality: $F(1,19)=4.64, p<.05 ; \mathrm{VF} \times$ Hemisphere $\times$ Anterior-Posterior: $F(3,57)=10.53, p<.01$. As expected, over the back of the head (where early visual potentials are most evident), stimuli presented to the LVF elicited slightly larger $\mathrm{N} 1$ components over right-hemisphere scalp sites $(\mathrm{RH}=$ $-0.35 \mu \mathrm{v}, \mathrm{LH}=-0.31 \mu \mathrm{v})$, whereas stimuli presented to the RVF elicited larger N1 components over left-hemisphere sites $(\mathrm{LH}=-1.06 \mu \mathrm{v}, \mathrm{RH}=0.06 \mu \mathrm{v}$.

$P 2(150-250 \mathrm{~ms})$. Figures 4 and 5 show ERPs from all scalp sites as a function of sentence type and visual field. In the P2 latency range, $\mathrm{VF}$ affected the topography of the ERPs, VF $\times$ Hemisphere: $F(1,19)=37.8, p<.0001$; VF $\times$ Hemisphere $\times$ Laterality: $F(1,19)=33.9, p<.0001 ; \mathrm{VF} \times$ Hemisphere $\times$ Anterior-Posterior $F(3,57)=11.8, p<.001$; VF $\times$ Hemisphere $\times$ Laterality $\times$ Anterior-Posterior: $F(3,57)=12.9, p<$ .01 . Over posterior electrodes, the crossover pattern observed in the $\mathrm{N} 1$ time window continued in this time interval. Over lateral frontal sites, the pattern in this time interval was similar to that observed in Experiment 1: LVF presentations elicited more negative responses than RVF presentations over right-scalp sites, whereas responses over left-scalp sites did not differ as a function of VF.

Congruity also modulated P2 amplitudes, with more positive responses to congruous than to incongruous items over frontal (especially right frontal) sites, Congruity $\times$ Hemisphere $\times$ Anterior-Posterior: $F(3,57)=3.61, p<.05$.

Although there were no overall effects of association on the P2, we did observe interactions among VF, association, and topo- graphic factors, $\mathrm{VF} \times$ Association $\times$ Hemisphere $\times$ AnteriorPosterior: $F(3,57)=3.29, p<.05$; VF $\times$ Association $\times$ Hemisphere $\times$ Laterality $\times$ Anterior-Posterior: $F(3,57)=3.0, p<.05$. We followed up on these interactions by measuring ERPs recorded at 11 frontal electrode sites (where P2 tends to be largest) within each VF. Analyses of both LVF and RVF data confirmed the presence of reliable congruity effects in both fields, $F s(1,19)>$ $4.8, p s<.05$, but failed to reveal association effects or interactions between association and the other factors. Thus the original interaction among VF, association, and topographic factors is likely to reflect the leading edge of the N400 rather than modulation of the $\mathrm{P} 2$ per se.

N400 (300-500 ms). In the peak latency range of the N400, lateralized presentation continued to influence scalp topography of the ERPs, evident in a main effect of VF, $F(1,19)=5.54, p<.05$, as well as interactions of VF with all the topographic factors, VF $\times$ Hemisphere: $F(1,19)=23 ., p<.0001$; VF $\times$ Anterior-Posterior: $F(3,57)=3.50, p<.05 ; \mathrm{VF} \times$ Hemisphere $\times$ Laterality: $F(1$, $19)=12.3, p<.01 ; \mathrm{VF} \times$ Hemisphere $\times$ Anterior-Posterior: $F(3,57)=18.7, p<.001 ; \mathrm{VF} \times$ Hemisphere $\times$ Laterality $\times$ Anterior-Posterior: $F(3,57)=6.22, p<.01$. This topography reflects the selection negativity described in Experiment 1, with larger negative potentials over lateral posterior electrode sites contralateral to the VF of presentation.

Figures 4 and 5 show that both varieties of incongruous sentence completion elicited much larger N400s than congruous completions, $F(1,19)=97.2, p<.0001$. This effect was broadly distributed but was largest over centroparietal scalp sites and was slightly larger over the right side of the head, as is typical of the visual N400 component, Congruity $\times$ Hemisphere: $F(1,19)=$ 25.8, $p<.001$; Congruity $\times$ Laterality: $F(1,19)=100.8, p<$ .0001 ; Congruity $\times$ Hemisphere $\times$ Anterior-Posterior: $F(3,57)=$ 9.77, $p<.001$; Congruity $\times$ Laterality $\times$ Anterior-Posterior: $F(3$, $57)=22.7, p<.0001$; Congruity $\times$ Hemisphere $\times$ Laterality $\times$ Anterior-Posterior: $F(3,57)=6.11, p<.01$. The congruity effect was independently significant for both visual fields, $F_{\mathrm{S}}(1,19)>$ 50.8, $p<.0001$. Although somewhat larger after RVF than after LVF presentations ( $3.8 \mu \mathrm{V}$ vs. $3.3 \mu \mathrm{V}$ ), there were no significant interactions involving VF and congruity in the omnibus ANOVA.

Figure 5 shows that, for RVF/lh presentations, lexical association reduced the amplitude of the N400 elicited by incongruous sentence completions. The lexical association effect was considerably smaller than the sentence congruity effect and was less broadly distributed across the scalp (evident only at centroparietal sites). This pattern of results led to no main effect of association but rather to a three-way Congruity $\times$ Association $\times$ Laterality interaction, $F(1,19)=4.62, p<.05$. In the congruous sentences alone, there was neither a main effect of association nor any interactions involving this factor. In the incongruous sentences alone, ERPs to associated words were slightly less negative than those to unassociated words, particularly over right scalp, Association $\times$ Hemisphere $\times$ Laterality, $F(1,19)=5.04, p<.05$.

\footnotetext{
${ }^{2}$ In fact, rather than decreased sensitivity due to noise, we found reliable experimental effects such as the association effect in congruous sentences discussed in the Experiment 2 Results N400 section (see Figures 4 and 5) in LVF ERPs that were absent from the RVF ERPs.
} 

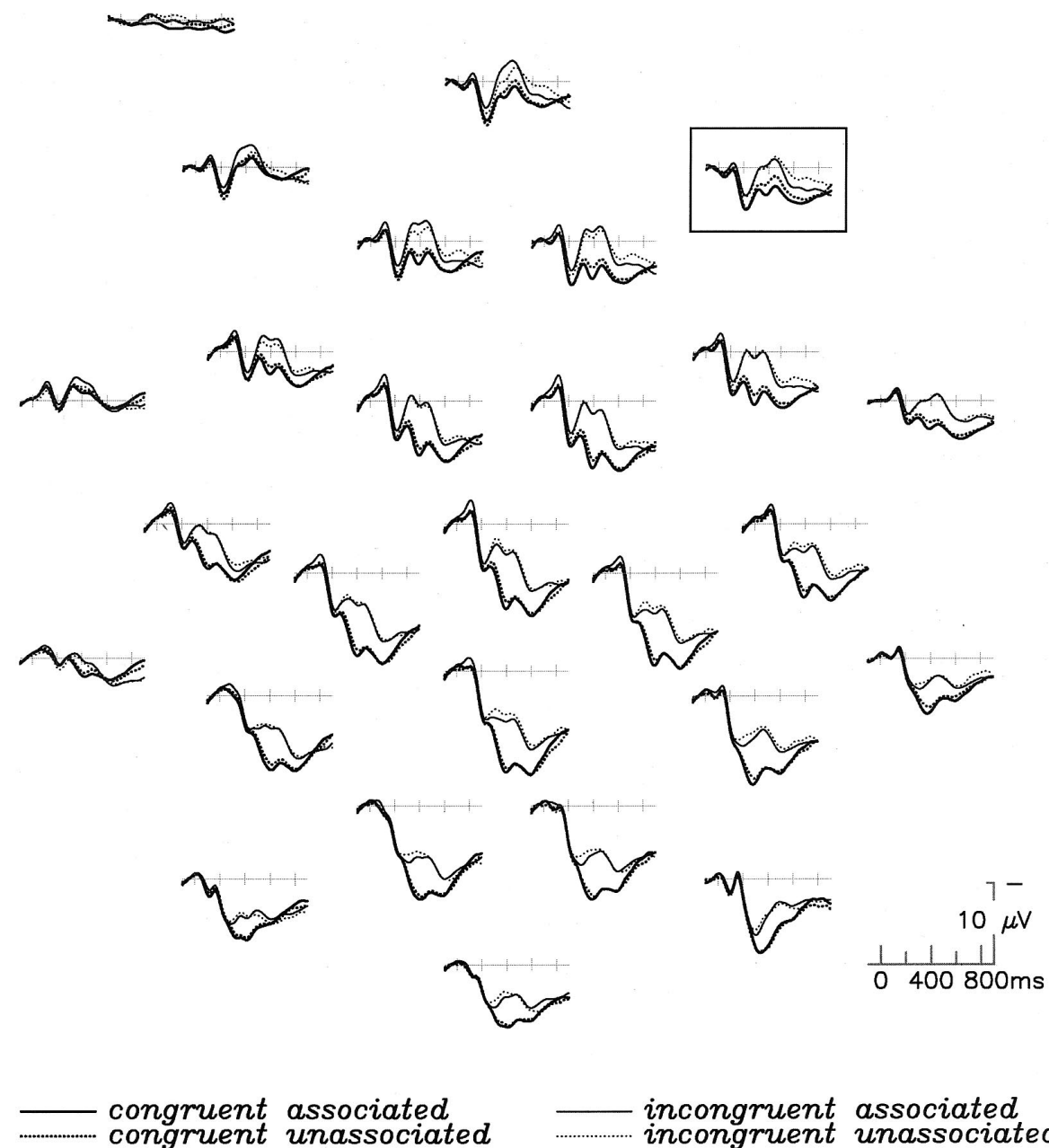
incongruent unassociated

Figure 4. Grand average event-related brain potentials (ERPs) to correctly named words in the left visual field in Experiment 2. Layout of electrode sites in the figure approximates their spatial location on the scalp, with midline prefrontal top and center and right occipital bottom right. Negative voltage is plotted up. The trace in the upper left corner is the electroocculogram, a bipolar montage of the electrical signal recorded at the side of the right eye, referenced to the side of the left eye. The boxed site highlights the focus of the association effect in the ERPs to congruous sentences.

Figure 4 shows that lexical association also influenced the ERPs elicited by LVF presentation, but that it did so in a qualitatively different manner than in the RVF conditions. Over centroparietal scalp, there was a small amplitude difference between the incongruous associated and incongruous unassociated conditions in the 300-500 ms range that did not reach statistical significance. (The 200-400 ms latency window was also measured, but it led to $F<$ 1 for the main effect of association and no significant interactions with association.) Instead, there was a small lexical association effect over right frontal scalp, apparent only for congruous sentences, leading to a Congruity $\times$ Association $\times$ Anterior-Posterior interaction, $F(3,57)=3.20, p<.05$. Follow-up analyses showed that this interaction was indeed attributable to a right frontal association effect in the congruous sentences alone, Association $\times$ Hemisphere $\times$ Anterior-Posterior: $F(3,57)=4.96, p<0.05$.

To assess whether N400 effects described above could be attributed to callosal transfer, we conducted two analyses designed to assess the timing of the congruity effect. First, we formed difference waves by subtracting the ERPs elicited by congruous endings from those elicited by incongruous endings (see Figure 6) Next, we measured the latency of the negative peak in the N400 interval and subjected the values to repeated measures ANOVAs with factors visual field (LVF or RVF) and scalp topography factors hemisphere, laterality, and anteriority. The N400 congruity effect peaked $411 \mathrm{~ms}(S D=51)$ after word onset with LVF presentation and $403 \mathrm{~ms}(S D=49)$ with RVF presentation. Analysis revealed neither a main effect of VF $(F<1)$ nor any interactions between $\mathrm{VF}$ and any of the topography factors (all $F$ s $<1.7$ ), suggesting that the peak latency of the N400 did not differ as a function of VF of presentation.

Figures 4 and 5 show that the sentence congruity effects began at about $200 \mathrm{~ms}$ after the onset of the final word. To assess the onset of the N400 congruity effect, we measured the latency at which congruity difference waves reached $10 \%$ of their peak 


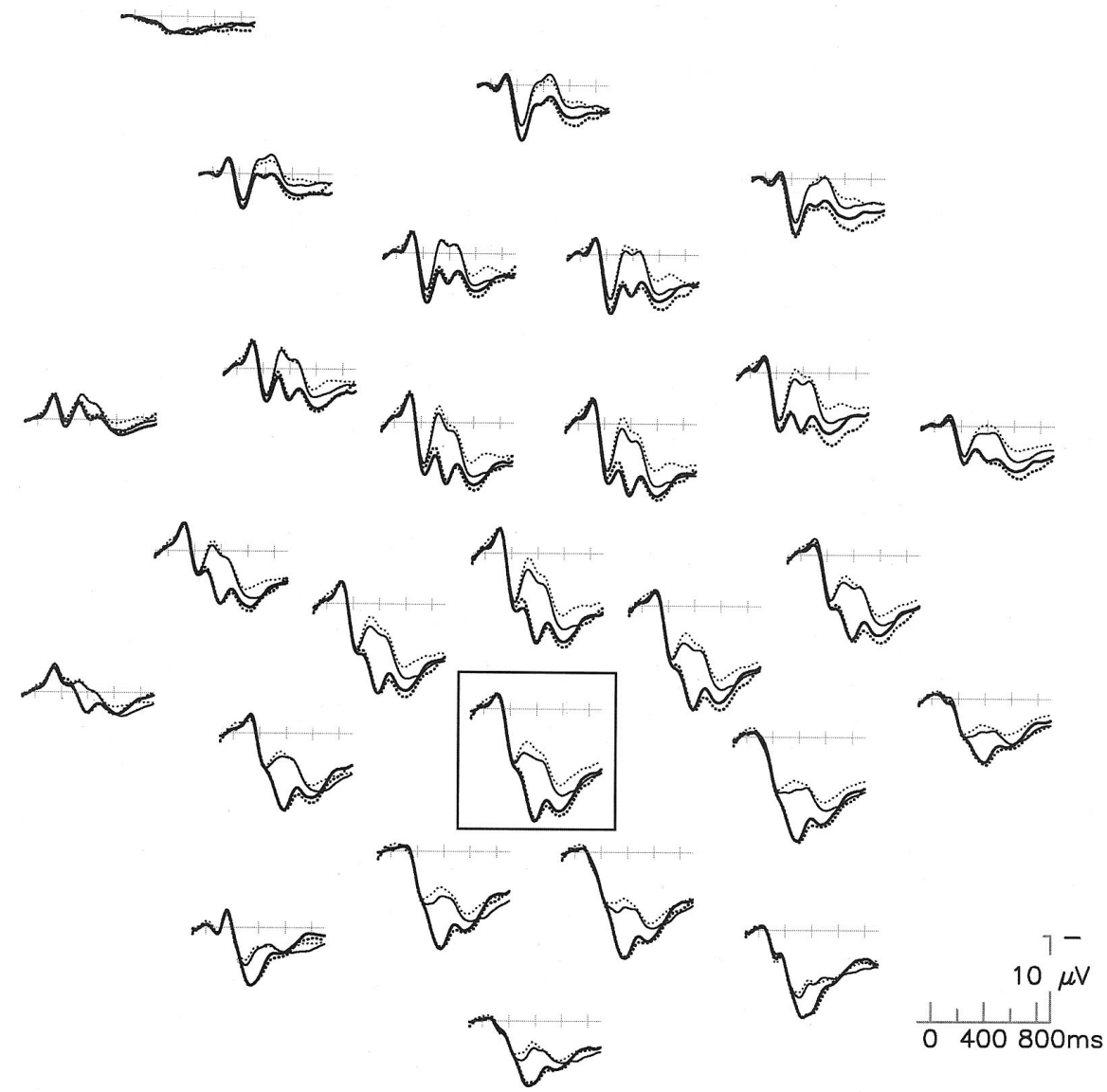

- congruent associated incongruent unassociated

Figure 5. Grand average event-related brain potentials (ERPs) to correctly named words in the right visual field in Experiment 2. Layout of electrode sites in the figure approximates their spatial location on the scalp, with midline prefrontal top and center and right occipital bottom right. Negative voltage is plotted up. The trace in the upper left corner is the electroocculogram, a bipolar montage of the electrical signal recorded at the side of the right eye, referenced to the side of the left eye. The boxed site highlights the focus of the association effect in the ERPs to incongruous sentences.

amplitude and subjected these values to analysis like that used on the peak latencies. Again, analyses suggested that VF of presentation did not affect the onset of the N400 effects $(F<1)$.

Late positive complex $(500-900 \mathrm{~ms})$. In the latest time window, side of presentation continued to influence the scalp topography of the ERPs; the waveforms were more negative over posterior scalp contralateral to the VF of presentation, selection negativity, reflected in VF $\times$ Hemisphere: $F(1,19)=14.1, p<$ .01 ; $\mathrm{VF} \times$ Anterior-Posterior: $F(3,57)=41.5, p<.0001$; $\mathrm{VF} \times$ Hemisphere $\times$ Laterality: $F(1,19)=17.7, p<.001 ; \mathrm{VF} \times$ Hemisphere $\times$ Anterior-Posterior: $F(3,57)=41.5, p<.0001$; $\mathrm{VF} \times$ Hemisphere $\times$ Laterality $\times$ Anterior-Posterior: $F(3,57)=$ $8.99, p<.001$.

The sentence congruity effect-more negative potentials for the incongruous completions-persisted into the late time window, main effect of congruity, $F(1,19)=6.11, p<.05$. When RVF and LVF responses were analyzed separately, both showed larger congruity effects over right scalp, Congruity $\times$ Hemisphere, $F(1$, $19)=5.96, p<.05$, for RVF; $F(1,19)=21.7, p<.001$, for LVF. This was particularly true for words presented to the LVF, resulting in a VF $\times$ Congruity $\times$ Hemisphere interaction in the omnibus ANOVA including both visual fields, $F(1,19)=6.39, p<.05$. The extended duration of the congruity effect after LVF presentation as compared with RVF presentation is most easily visualized in Figure 6, showing difference waves we formed by subtracting the ERPs elicited by congruous endings from those elicited by incongruous endings.

The omnibus ANOVA yielded two interactions for the lexical association manipulation: Congruity $\times$ Association, $F(1,19)=$ 4.60, $p<.05$, and a complex VF $\times$ Association $\times$ Hemisphere $\times$ Laterality interaction, $F(1,19)=4.39, p<.05$. Figure 5 shows that the RVF results were responsible for the Congruity $\times$ Association interaction: In incongruous sentences, the associated words elicited more positive potentials, continuing the pattern observed 


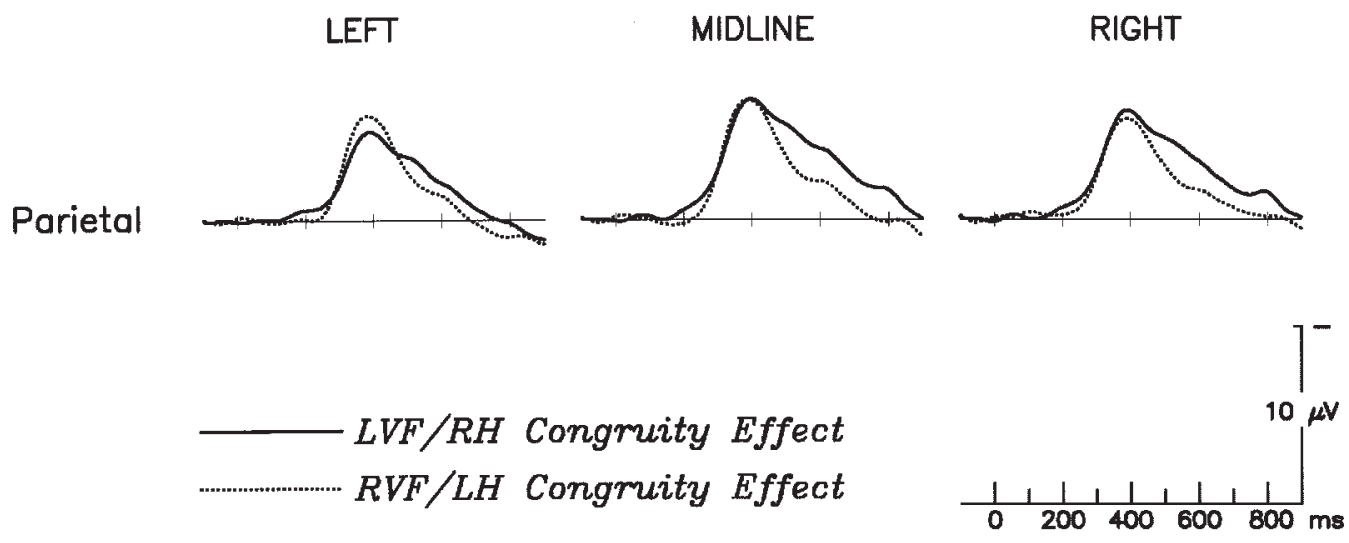

Figure 6. Difference waves showing the sentence congruity effects at three midline parietal scalp sites (in the sixth row of scalp electrodes in Figures 4 and 5) in Experiment 2, for final words presented in the right versus left visual fields. Negative voltage is plotted up. $\mathrm{LVF}=$ left visual hemifield; $\mathrm{RH}=$ right hemisphere; $\mathrm{RVF}=$ right visual hemifield; $\mathrm{LH}=$ left hemisphere.

during the N400 epoch but evident at almost all scalp sites. This observation was confirmed via separate analysis of the incongruous words in the RVF association, $F(1,19)=8.62, p<.01$. In congruous sentences, the impact of association in the RVF was much smaller and resulted in no significant main effect or interactions involving association.

Figure 4 shows that the LVF results were responsible for the second interaction in the omnibus ANOVA. Although there was no evident impact of association over much of the scalp, more positive potentials were elicited by associated words over prefrontal and frontal scalp, especially on the right side (the same general location for the LVF association effects in the N400 latency range). Analysis of the LVF data alone thus yielded an Association $\times$ Hemisphere $\times$ Laterality interaction, $F(1,19)=4.71, p<$ .05. For the LVF, the slightly enhanced positive potential for associated words was similar in congruous and incongruous sentence completions. However, the association effect in the LVF was too small to be independently significant when the congruous and incongruous sentences were analyzed separately.

Summary. As in Experiment 1, lateralized presentation of the stimuli resulted in larger contralateral N1 responses and an extended contralateral selection negativity over posterior electrode sites. Sentential congruity affected the ERP response beginning around $200 \mathrm{~ms}$ and continuing to about $900 \mathrm{~ms}$ after stimulus onset. In all time windows, responses were more positive to congruous than to incongruous completions. Congruity effects did not reliably differ in size or onset latency as a function of VF but were more prolonged after LVF/rh presentation. Small, spatially restricted effects of association were also evident on the N400 and LPC. With RVF/lh presentation, N400 and LPC association effects were confined to incongruous sentences. With LVF presentation, N400 association effects were reliable only in congruous sentences, whereas LPC association effects were marginal but did not differ as a function of sentence congruity.

\section{Discussion}

The results of Experiment 2 strongly suggest that both hemispheres are primarily driven by message-level congruity during sentence reading. Regardless of VF of presentation, ERPs to lateralized targets revealed large, robust effects of sentence congruity on the P2, N400, and LPC and smaller effects of association on the N400 and LPC. VF of presentation did not reliably modulate the congruity effect in the P2 or the N400 intervals, although the congruity $\mathrm{N} 400$ was slightly larger with $\mathrm{RVF} / \mathrm{lh}$ presentation. The LPC congruity effect, however, was larger with LVF/rh presentation. These data thus demonstrate that both hemispheres use message-level congruity information to shape the real-time processing of words in sentence contexts.

Overall association effects were also observed on the N400 extending into the interval of the LPC. Though much smaller and less broadly distributed than the congruity effects described above, unassociated stimuli elicited more negative (less positive) ERPs than did associated stimuli from 300 to $900 \mathrm{~ms}$ postonset. With $\mathrm{RVF} / \mathrm{lh}$ presentation, association effects were confined to incongruous sentences both during the N400 and LPC. With LVF/rh presentation, by contrast, association effects were more reliable for congruous sentences during the N400 and did not differ as a function of congruity during the LPC. Thus, whereas the LH used word-level context only when sentence-level context was not supportive (i.e., only in incongruous sentences), the RH displayed a preserved sensitivity to word-level context in congruous sentences, where it was somewhat redundant. These differential patterns suggest that, although both hemispheres use message-level information, they do so in different ways that make different use(s) of word-level information.

\section{General Discussion}

\section{Lexical and Sentential Context Mechanisms}

In Experiment 1, ERPs were recorded from healthy adults as they read associated and unassociated word pairs. With both LVF/rh and RVF/lh presentation, associated words elicited more positive ERPs in the P2, N400, and LPC time windows. Consistent with reports using behavioral measures of hemifield priming (Chiarello, 1998; Chiarello et al., 2003; Coney, 2002), these ERP 
data suggest that both hemispheres are able to benefit from the prior occurrence of a strongly associated word.

In Experiment 2, the associated and unassociated word pairs from Experiment 1 were put into sentence contexts, where the targets formed congruous or incongruous sentence endings (see Table 2). ERPs were recorded as neurologically intact participants read these sentences. As evident in Figure 7, the presence of a sentential context resulted in a dramatic attenuation of the association effect on the ERPs. The same word-pair relationship that elicited a broadly distributed $3 \mu \mathrm{V} \mathrm{N} 400$ effect in Experiment 1 elicited a spatially limited N400 effect that was less than $1 \mu \mathrm{V}$ in amplitude in Experiment 2. Instead, sentence congruity was the chief determinant of N400 amplitude in Experiment 2; congruity effects in Experiment 2 were similar in size to those observed for association in Experiment 1. These findings suggest that lexical context mechanisms were readily employed when words appeared in word pairs but had remarkably little influence on processing when the same word pairs appeared in sentence contexts.

Prior work has shown that under some circumstances, lexical and sentence-level context can exert additive effects on ERP measures. For instance, Van Petten (1993) examined associated and unassociated word pairs embedded in either normal sentences (associated, When the moon is full it is hard to see many stars or the Milky Way. and unassociated, When the insurance investigators found out that he'd been drinking they refused to pay the claim.) or fully anomalous sentences (associated, When the moon is rusted it is available to buy many stars or the Santa Ana. And unassociated, When the insurance supplies explained that he'd

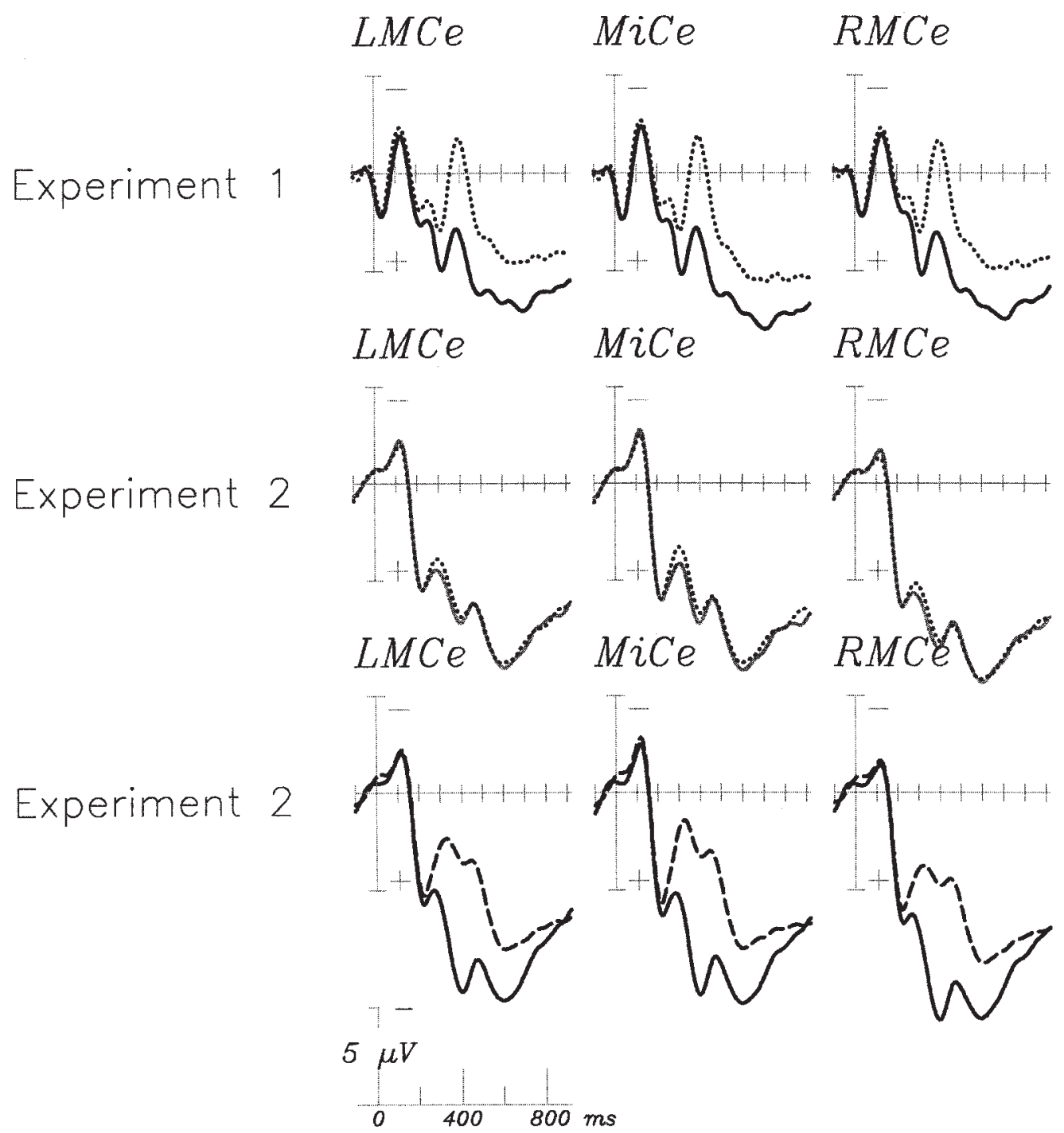

Figure 7. Summary of the semantic context effects across the two experiments: Grand average event-related brain potentials from a midline parietal scalp site (the middle trace in the ninth row of scalp electrodes in Figures 4 and 5). Negative voltage is plotted up. In the first two rows, dotted lines indicate unassociated stimuli, and solid lines indicate associated stimuli; in the third row, dashed lines indicate incongruous stimuli, and solid lines indicate congruous stimuli. $\mathrm{LMCe}=$ left medial central; $\mathrm{MiCe}=$ midline central; $\mathrm{RMCe}=$ right medial central. 
been complaining they refused to speak the keys.). This design made it possible to compare the respective contributions of lexical association and a supportive sentential context with N400 amplitude. The results suggested that both factors independently influenced the N400 - that is, in the normal associated sentences, both lexical and sentential context were exploited to facilitate processing. The additivity pattern has been replicated with both printed and spoken materials in healthy young adults and also for spoken sentences in older adults and Alzheimer's patients, who are less able to exploit the message-level information and correspondingly get an added boost from association (Schwartz et al., 2003; Van Petten, 1993; Van Petten et al., 1997).

A key difference between the Van Petten (1993) materials and those of the present study, however, is in the strength of the context information available at the target (associated or unassociated) word. Whereas the prior studies looked at relatively weakly constrained sentence-intermediate words, in this study, we examined responses to fairly highly constrained sentence-final words. Our congruous associated and congruous unassociated sentences were matched for overall predictability (operationalized as cloze probability), so that word association added little or no information to that derived from message-level constraints. Further, in the incongruous sentences, the two types of context (lexical and sentential) were pitted against each other. Results of this study suggest that when sentence-level context provides the critical information, it largely overrides word association.

Although the related word pairs produced strong semantic effects in isolation, the differences between associated and unassociated pairs became quite small when they were placed in sentence contexts. This result is much like that observed with central presentation of the materials (Van Petten et al., 1999). Similar to patterns observed for word frequency (Van Petten \& Kutas, 1990), then, it seems that word-association information can facilitate processing when message-level information is absent (as in Experiment 1 of this study), weakly supportive (as in Van Petten, 1993), and in participants with reduced access to message-level information (as in Schwartz et al., 2003). However, when, as in Experiment 2 of this study, strong message-level information eclipses word-level information, the latter has little impact on processing.

\section{Hemifield Presentation}

What, then, of the hypothesis that the RH is sensitive to wordlevel context, whereas the $\mathrm{LH}$ is sensitive to both word and sentence-level context? Before considering this issue, we briefly review the results that indicate that the hemifield presentation employed in this study was successful in shifting the balance of stimulus processing between the two cerebral hemispheres.

In both experiments, the N1 component was larger over the hemisphere contralateral to the visual field of presentation. Following the N1, a similarly contralateral negative potential (selection negativity) extended throughout the recording epoch, indicating that at least some aspects of neural activity continued to depend on the initial VF. These two ERP results replicate prior studies using the hemifield presentation paradigm (Federmeier \& Kutas, 1999, 2001). The temporally latest outcome (i.e., the one observed $2.5 \mathrm{~s}$ after stimulus offset) of the hemifield manipulation was reduced accuracy in naming words presented in the LVF, also a standard result (Chiarello, 1988, 1998). Unnameable words were, however, eliminated from the ERP data to focus on semantic processing per se rather than on perceptual-level differences in the hemispheres' ability to decode text (Jordan, Thomas, \& Patching, 2003).

Although these three results indicate a pervasive impact of the hemifield manipulation, it would be untenable to argue that words presented to one VF were processed solely by the contralateral hemisphere. The participants were neurologically healthy with intact cerebral commissures allowing transmission of information between the two hemispheres. After some initial processing by unilateral visual cortex, one would expect information from one VF to be distributed to both hemispheres. However, one aspect of the results clearly indicates that the language-dominant left hemisphere was not solely responsible for the observed semantic context effects. The supposition that LVF stimuli can be semantically processed only after transmission to the left hemisphere predicts a latency delay (typically $25-40 \mathrm{~ms}$ ) relative to RVF stimuli. However, in Experiment 1, the onset and the peak of the N400 association effect was similar in the VFs, though the effect itself was slightly larger with RVF presentation. Likewise, in Experiment 2, there was no difference in the onset and peak latencies of the sentence congruity effect after RVF versus LVF presentation (Figure 6; see Federmeier \& Kutas, 1999, for a similar null effect). Overall, multiple aspects of the results suggest that LVF presentation resulted in a greater degree of right-hemisphere processing than RVF presentation and vice versa.

\section{Message-Blind RH Model}

As noted above, one model of functional asymmetry in language comprehension posits a fundamental difference in the abilities of the two hemispheres to exploit message-level information (Chiarello, 2000; Faust, 1998; Faust et al., 1993, 1995; Faust \& Gernsbacher, 1996). In this message-blind RH model, the left hemisphere constructs a message-level representation by integrating syntactic, semantic, and pragmatic information, whereas the right hemisphere is primarily capable of only bottom-up semantic activations prompted by incoming words. The message-blind $\mathrm{RH}$ model predicted different patterns of ERP effects depending on hemifield of presentation. In particular, LVF presentation would be expected to give rise to association effects but no congruity effects. This was not the case. Presentation to both visual fields gave rise to large and robust effects of sentence congruity. Moreover, the size of the congruity effects dwarfed the association effects with presentation to either visual field (Figure 7).

The results of this study are therefore in conflict with earlier work that failed to detect context sensitive responses with presentation to the LVF/rh. For example, Faust et al. (1995) found sentence congruity effects only with RVF/lh presentation. However, Chiarello et al., (2001) pointed out that the incongruous sentences used in this earlier work became incongruous almost immediately, which may have induced an unnatural processing set among participants. Likewise, other studies that support the message-blind RH model (Chiarello, Liu, \& Faust, 1999; Faust, 1998) have been limited by methodological shortcomings (see Chiarello et al., 2001 for details).

Our results are, however, consistent in some ways with those of a similarly motivated study by Chiarello et al., (2001; see also 
Faust et al., 2003). In their study, low-constraint sentences ended either congruously or incongruously and either did or did not contain a lexical associate of the laterally presented target word. As in the present study, both RVF and LVF presentation produced congruity effects, suggesting that both hemispheres were sensitive to some aspects of sentence-level context. However, priming for congruous completions was observed only when the sentence also contained a lexical associate of the target (final) word (Chiarello et al., 2001). Their results are thus ambiguous as to whether the observed priming effects reflect word-level context, sentence-level context, or some combination of both. The present study is thus unique in demonstrating congruity effects with presentation to the LVF in sentences that contained a lexical associate of the target, and, more importantly, also in those lacking lexically associated words.

The results of the present study are consistent with other ERP studies using hemifield presentation. One such study compared expected and unexpected completions of brief paragraphs, such as They wanted to make the hotel look more like a tropical resort. So, all along the driveway, they planted rows of palms/tulips. The outcome was an N400 congruity effect of similar magnitude after RVF and LVF presentation (Federmeier \& Kutas, 1999). A recent study of joke comprehension similarly led to equivalent effects of cloze probability with RVF and LVF presentation (Coulson \& Williams, 2005). In these studies, the equivalent sentence congruity effects across visual fields were accompanied by hemifield differences in the brain response to category structure and to the cognitive aspects of joke comprehension, indicating that the hemifield method is capable of revealing processing asymmetries.

Our suggestion that context-sensitivity characterizes both hemispheres is also consistent with the finding that patients with damage to either hemisphere display deficits in their ability to exploit sentence-level context to determine the appropriate meaning of ambiguous words, suggesting that mechanisms in both cerebral hemispheres contribute to sensitivity to higher level context (Grindrod \& Baum, 2003). ERP studies of aphasic patients have indicated that the ability to use sentence-level context in lexical integration is preserved with LH damage, though it is delayed (Swaab, Brown, \& Hagoort, 1997, 1998). The ability of aphasic patients to use contextual information may reflect the contribution of their intact RH. Finally, RH sensitivity to message-level context is more congruent with findings from neuropsychology that RHD results in an impaired ability to appreciate context-dependent aspects of meaning important for figurative language comprehension (Joanette et al., 1990).

\section{Sentential and Lexical Context: Differences Between the Hemispheres}

The stimulus materials were designed so that lexically associated word pairs could blend seamlessly into a sentence-level representation in congruous sentences but would conflict with the sentence-level message in incongruous sentences. For presentation to either visual field, this is largely what happened, with the sentence-level representation proving dominant (Figure 7). However, small effects of lexical association did emerge in brain activity, and these took different forms for the two visual fields. After presentation to the RVF/lh, no impact of lexical association was reliable for congruous sentences, but lexically related words in the RVF were able to reduce the amplitude of the large N400 normally elicited by incongruous sentence completions (Figure 5). After the peak of the N400, a spatially widespread positive potential (LPC) was enhanced by associated as compared with unassociated words in incongruous sentences. This pattern of results might be expected from a processor that is strongly biased toward sentential context but is additionally sensitive to other sources of context when sentence integration fails.

After presentation to the $\mathrm{LVF} / \mathrm{rh}$, smaller effects of lexical association were observed than in the RVF, indicating a generally weaker ability to use this source of semantic context. These effects differed in scalp topography: The RVF effects occurred at the centroparietal sites at which semantic context effects are typically maximal in ERPs (Kutas, Van Petten, et al., 1988), whereas the LVF effects were most evident at right frontal sites (Figures 4 and 5). The functional significance of this difference awaits explication in future research. A final, critical, difference is that association effects were observed in congruous sentences only with presentation to the LVF. These effects began in the latency range of the N400 and continued through the LPC time window, where there were also significant effects in incongruous sentences.

The presence of LVF lexical association effects in congruous sentences might suggest greater $\mathrm{RH}$ reliance on word-level relationships in the lexical integration processes indexed by the N400. Federmeier and Kutas $(1999,2002)$ have hypothesized that only the LH uses sentence context information to actively prepare for the processing of likely upcoming words-in other words, to predict. The $\mathrm{RH}$, in contrast, is hypothesized to use an integration strategy that compares the features of the obtained word with those of the context. This RH comparison process might be more likely than the predictive strategy to use word-level relationships between a target and a context word in a congruent sentence, consistent with the pattern reported herein.

Besides differences in the impact of word-level context in congruous and incongruous sentences, hemifield of presentation also had subtle effects on the temporal progression of the semantic effects. In Experiment 1, for example, the LVF word-association effect was somewhat smaller than that in the RVF when measured 300-500 ms but was slightly larger than the RVF effect in the 600-800 ms measurement interval. The same tradeoff between the two latency windows was observed for the sentence congruity effects in Experiment 2. Although similar effect onset latencies in both VFs would allow one to argue against marked hemispheric differences in the onset of semantic activation, the observed pattern of timing results is consistent with the claim that word processing in the right hemisphere is somewhat slow and prolonged relative to the left hemisphere (Burgess \& Lund, 1988; Burgess \& Simpson, 1998).

Irrespective of mechanism, the results support the conclusion that the two hemispheres both exploit word- and sentence-level context in the processing of a current word, and weight the use of this information by its relative usefulness. When only lexical relations are present, as in the word pairs of Experiment 1, these strongly influence processing. When both forms of context are available, sentence-level information is weighted more heavily, but the two hemispheres showed differential reliance on lexical relations in sentence contexts. For the left hemisphere, lexical relationships appear to be secondary and influential only when sentence integration fails. For the right hemisphere, lexical rela- 
tionships influence processing even in congruous sentence contexts. It seems likely that observed hemispheric asymmetries in the weighting of word and sentence-level information-as well as possible differences in semantic activation and integration processes-are what allow the brain as a whole to process language in an astonishingly efficient yet flexible manner.

\section{References}

Beeman, M. J., \& Chiarello, C. (1998). Complementary right- and lefthemisphere language comprehension. Current Directions in Psychological Science, 7(1), 2-8.

Bentin, S. (1987). Event-related potentials, semantic processes, and expectancy factors in word recognition. Brain \& Language, 31, 308-327.

Bub, D. N., \& Arguin, M. (1995). Visual word activation in pure alexia. Brain and Language, 36, 357-376.

Burgess, C., \& Lund, K. (1998). Modeling cerebral asymmetries in highdimensional space. In M. Beeman \& C. Chiarello (Eds.), Right hemisphere language comprehension: Perspectives from cognitive neuroscience (pp. 215-244). Mahwah, NJ: Erlbaum.

Burgess, C., \& Simpson, G. (1988). Cerebral hemispheric mechanisms in the retrieval of ambiguous word meaning. Brain and Language, 42, 203-217.

Chiarello, C. (1988). Lateralization of lexical processes in the normal brain: A review of visual half-field research. In H. H. Whitaker (Ed.), Contemporary reviews in neuropsychology (pp. 59-69). New York: Springer-Verlag.

Chiarello, C. (1991). Interpretation of word meanings by the cerebral hemispheres: One is not enough. In P. Schwanenflugel (Ed.), The psychology of word meaning. Hillsdale, NJ: Erlbaum.

Chiarello, C. (1998). On codes of meaning and the meaning of codes: Semantic access and retrieval within and between hemispheres. In C. C. M. Beeman (Ed.), Right hemisphere language comprehension: Perspectives from cognitive neuroscience (pp. 141-160). Mahwah, NJ: Erlbaum.

Chiarello, C. (2000). Inferring the nature of semantic processes by varying priming procedure: A reply to Koivisto and Laine. Laterality, 5, 23-27.

Chiarello, C., Burgess, C., Richards, L., \& Pollock, A. (1990). Semantic and associative priming in the cerebral hemisphere: Some words do, some words don't . . . sometimes, some places. Brain and Language, 38, $75-104$

Chiarello, C., Liu, S., \& Faust, M. (1999). Cerebral asymmetries in sentence priming and the influence of semantic anomaly. Brain and Cognition, 40(1), 75-79.

Chiarello, C., Liu, S., \& Faust, M. (2001). Bihemispheric sensitivity to sentence anomaly. Neuropsychologia, 39(13), 1451-1463.

Chiarello, C., Liu, S., Shears, C., Quan, N., \& Kacinik, N. (2003). Priming of strong semantic relations in the left and right visual fields: A timecourse investigation. Neuropsychologia, 41, 721-732.

Coltheart, M. (1981). The MRC psycholinguistic database. Quarterly Journal of Experimental Psychology: Human Experimental Psychology, 33, 497-505.

Coney, J. (2002). The effect of associative strength on priming in the cerebral hemispheres. Brain and Cognition, 50, 234-241.

Coulson, S., \& Williams, R. W. (2005). Hemispheric asymmetries and joke comprehension. Neuropsychologia, 43, 128-141.

Dale, A. M., \& Sereno, M. I. (1993). Improved localization of cortical activity by combining EEG and MEG with MRI cortical surface reconstruction: A linear approach. Journal of Cognitive Neuroscience, 5, $162-176$.

Dunn, B. R., Dunn, D. A., Languis, M., \& Andrews, D. (1998). The relation of ERP components to complex memory processing. Brain Cognition, 36(3), 355-376.

Faust, M. (1998). Obtaining evidence of language comprehension from sentence priming. In M. Beeman \& C. Chiarello (Eds.), Right hemisphere language comprehension: Perspectives from cognitive neuroscience (pp. 161-186). Hillsdale, NJ: Erlbaum.

Faust, M., Babkoff, H., \& Kravets, S. (1995). Linguistic processes in the two cerebral hemispheres: Implications for modularity vs. interactionism. Journal of Clinical and Experimental Neuropsychology, 17, 171192.

Faust, M., Bar-lev, A., \& Chiarello, C. (2003). Sentence priming effects in the two cerebral hemispheres: Influences of lexical relatedness, word order, and sentence anomaly. Neuropsychologia, 41, 480-492.

Faust, M., \& Gernsbacher, M. (1996). Cerebral mechanisms for suppression of inappropriate information during sentence comprehension. Brain and Language, 53, 234-259.

Faust, M., Kravetz, S., \& Babkoff, H. (1993). Hemisphericity and topdown processing of language. Brain and Language, 53, 234-259.

Federmeier, K. D., \& Kutas, M. (1999). Right words and left words: Electrophysiological evidence for hemispheric differences in meaning processing. Cognitive Brain Research, 8, 373-392.

Federmeier, K. D., \& Kutas, M. (2001). Meaning and modality: Influences of context, semantic memory organization, and perceptual predictability on picture processing. Journal of Experimental Psychology: Learning, Memory, and Cognition, 27, 202-224.

Federmeier, K. D., \& Kutas, M. (2002). Picture the difference: Electrophysiological investigations of picture processing in the two cerebral hemispheres. Neuropsychologia, 40, 730-747.

Francis, W., \& Kučera, H. (1982). Frequency analysis of English usage. New York: Houghton Mifflin.

Gazzaniga, M. S., \& Sperry, R. W. (1967). Language after section of the cerebral commissures. Brain, 90, 131-138.

Grindrod, C. M., \& Baum, S. R. (2003). Sensitivity to local sentence context information in lexical ambiguity resolution: Evidence from leftand right-hemisphere-damaged individuals. Brain and Language, 85, 503-523.

Hagoort, P., Brown, C., \& Swaab, T. Y. (1996). Lexical-semantic eventrelated potential effects in patients with left hemisphere lesions and aphasia, and patients with right hemisphere lesions without aphasia. Brain, 119, 627-649.

Halgren, E., Dhond, R. P., Christensen, N., Van Petten, C., Marinkovic, K., Lewine, J. D., et al. (2002). N400-like MEG responses modulated by semantic context, word frequency, and lexical class in sentences. $\mathrm{Neu}$ roimage, $17(3), 1101-1116$.

Hellige, J. (Ed.). (1983). Cerebral hemisphere asymmetry: Method, theory, and application. New York: Praeger.

Hillyard, S., \& Anllo-Vento, L. (1998). Event-related brain potentials in the study of visual selective attention. Proceedings of the National Academy of Sciences of the United States of America, 95, 781-787.

Holcomb, P. (1988). Automatic and attentional processing: An eventrelated brain potential analysis of semantic priming. Brain and Language, 35, 66-85.

Hopf, J.-M., Vogel, E., Woodman, G., Heize, H.-J., \& Luck, S. J. (2002). Localizing visual discrimination processes in time and space. Journal of Neurophysiology, 88, 2088-2095.

Joanette, Y., Goulet, P., \& Hannequin, D. (1990). Right hemisphere and verbal communication. New York: Springer-Verlag.

Jordan, T. R., Thomas, S. M., \& Patching, G. R. (2003). Assessing the importance of letter pairs in reading-parafoveal processing is not the only view: Reply to Inhoff, Radach, Eiter, and Skelly (2003). Journal of Experimental Psychology: Learning, Memory, and Cognition, 29, $900-$ 903.

King, J. W., Ganis, G., \& Kutas, M. (1998). Potential asymmetries in language comprehension: In search of the electrical right. In M. J. Beeman \& C. Chiarello (Eds.), Right hemisphere language comprehension: Perspectives from cognitive neuroscience. Hillsdale, NJ: Erlbaum. Kiss, G. R., Armstrong, C., Milroy, R., \& Piper, J. (1973). An associative 
thesaurus of English and its computer analysis. In A. J. Aitken, R. W. Bailey, \& N. Hamilton-Smith (Eds.), The computer and literary studies (pp. 271-286). Edinburgh, Scotland: Edinburgh University Press.

Koivisto, M., Laine, M. (2000). Hemispheric asymmetries in activation and integration of categorical information. Laterality, 5, 1-21.

Kuperberg, G., McGuire, P. K., Bullmore, E. T., Brammar, M. J., RabeHesketh, S., Wright, I. C., et al. (2000). Common and distinct neural substrates for pragmatic, semantic, and syntactic processing of spoken sentences: An fMRI study. Journal of Cognitive Neuroscience, 12, 321-341.

Kutas, M. (1993). In the company of other words: Electrophysiological evidence for single-word and sentence context effects. Language and Cognitive Processes, 8, 533-572.

Kutas, M., Federmeier, K. D., Coulson, S., King, J., \& Munte, T. F. (2000). Language. In J. T. Cacioppo, L. G. Tassinary, \& G. Berntson (Eds.), Handbook of psychophysiology (2nd ed., pp. xiii, 1039). Cambridge, England: Cambridge University Press.

Kutas, M., \& Hillyard, S. A. (1980). Reading senseless sentences: Brain potentials reflect semantic incongruity. Science, 207, 203-205.

Kutas, M., Hillyard, S. A., \& Gazzaniga, M. S. (1988). Processing of semantic anomaly by right and left hemispheres of commissurotomy patients: Evidence from event-related brain potentials. Brain, 111, 553576.

Kutas, M., \& Van Petten, C. (1994). Psycholinguistics electrified. In M. Gernsbacher (Ed.), Handbook of psycholinguistics (pp. 83-143). San Diego, CA: Academic Press.

Kutas, M., Van Petten, C., \& Besson, M. (1988). Event-related potential asymmetries during the reading of sentences. Electroencephalography and Clinical Neurophysiology, 69, 218-233.

Luck, S. J., \& Hillyard, S. A. (1994). Spatial filtering during visual search: Evidence from human electrophysiology. Journal of Experimental Psychology: Human Perception and Performance, 20, 1000-1014.

McDonald, S. (1996). Clinical insights into pragmatic theory: Frontal lobe deficits and sarcasm. Brain and Language, 68, 486-506.

Meyer, D.E., \& Schvaneveldt, R.W. (1971). Facilitation in recognizing pairs of words: Evidence of a dependence between retrieval operations. Journal of Experimental Psychology, 90, 227-234.

Neville, H. J., Kutas, M., \& Schmidt, A. (1982). Event-related potential studies of cerebral specialization during reading: I. Studies of normal adults. Brain and Language, 16, 300-315.

Nobre, A. C., \& McCarthy, G. (1995). Language-related field potentials in the anterior-medial temporal lobe: II. Effects of word type and semantic priming. Journal of Neuroscience, 15(2), 1090-1098.

Osterhout, L., \& Holcomb, P. (1995). Event related potentials and language comprehension. In M. D. Rugg \& M. G. H. Coles (Eds.), Electrophysiology of mind: Event-related brain potentials and cognition (pp. 171215). Oxford, England: Oxford University Press.

Rugg, M. D., \& Coles, M. (Eds.). (1995). Electrophysiology of mind:
Event-related brain potentials and cognition. Oxford, England: Oxford University Press.

Schwartz, T. J., Federmeier, K. D., Van Petten, C., Salmon, D. P., \& Kutas, M. (2003). Electrophysiological analysis of context effects in Alzheimer's disease. Neuropsychology, 17(2), 187-201.

Swaab, T. Y., Brown, C., \& Hagoort, P. (1997). Spoken sentence comprehension in aphasia: Event-related potential evidence for a lexical integration deficit. Journal of Cognitive Neuroscience, 9, 39-66.

Swaab, T. Y., Brown, C., \& Hagoort, P. (1998). Understanding ambiguous words in sentence contexts: Electrophysiological evidence for delayed contextual selection in Broca's aphasia. Neuropsychologia, 36(8), 737761.

Titone, D. (1998). Hemispheric differences in context sensitivity during lexical ambiguity resolution. Brain and Language, 65, 361-394.

Urbach, T., \& Kutas, M. (2002). The intractability of scaling scalp distributions to infer neural sources. Psychophysiology, 39, 791-808.

Van Petten, C. (1993). A comparison of lexical and sentence-level context effects in event-related potentials. Language and Cognitive Processes, 8 , 485-531.

Van Petten, C. (1995). Words and sentences: Event-related brain potential measures. Psychophysiology, 32(6), 511-525.

Van Petten, C., Coulson, S., Weckerly, J., Federmeier, K. D., Folstein, J., \& Kutas, M. (1999). Lexical association and higher-level semantic context: An ERP Study. Journal of Cognitive Neuroscience Supplement, 46.

Van Petten, C., \& Kutas, M. (1990). Interactions between sentence context and word frequency in event-related brain potentials. Memory \& Cognition, 18, 380-393.

Van Petten, C., Kutas, M., Kluender, R., Mitchiner, M., \& McIsaac, H. (1991). Fractionating the word repetition effect with event-related potentials. Journal of Cognitive Neuroscience, 3, 131-150.

Van Petten, C., \& Rheinfelder, H. (1995). Conceptual relationships between spoken words and environmental sounds: Event-related brain potential measures. Neuropsychologia, 33, 485-508.

Van Petten, C., Weckerly, J., McIsaac, H., \& Kutas, M. (1997). Working memory capacity dissociates lexical and sentential context effects. Psychological Science, 8, 238-242.

Zaidel, E. (1983). Disconnection syndrome as a model for laterality effects in the normal brain. In J. Hellige (Ed.), Cerebral hemisphere asymmetry: Method, theory and application (pp. 95-151). New York: Praeger.

Zaidel, E. (1990). Language functions in the two hemispheres following complete cerebral commisurotomy and hemispherectomy. In F. Boller \& J. Graffman (Eds.), Handbook of neuropsychology (Vol. 4, pp. 115150). New York: Elsevier.

Received January 6, 2004

Revision received July 8, 2004

Accepted July 26, 2004 